\title{
Diterpene and other Constituents from Stemodia maritima (Scrophulariaceae)
}

\author{
Francisco E. A. Rodrigues, ${ }^{a}$ Jefferson Q. Lima ${ }^{a, b}$ Maria da Conceição F. de Oliveira, ${ }^{a}$ \\ Jackson N. Vasconcelos, ${ }^{a}$ Gilvandete M. P. Santiago, ${ }^{a, c}$ Jair Mafezoli, ${ }^{a}$ \\ Raimundo Braz-Filho ${ }^{d}$ and Angela M. C. Arriaga*,a
}

\author{
${ }^{a}$ Curso de Pós-Graduação em Química, Departamento de Química Orgânica e Inorgânica, \\ Universidade Federal do Ceará, CP 6036, 60451-970 Fortaleza-CE, Brazil \\ ${ }^{b}$ Curso de Engenharia Ambiental, Instituto Federal do Ceará, Campus Juazeiro do Norte, \\ 63040-000 Juazeiro do Norte-CE, Brazil \\ 'Departamento de Farmácia, Universidade Federal do Ceará, 60430-370 Fortaleza-CE, Brazil \\ ${ }^{d}$ Centro de Ciências e Tecnologias, Universidade Estadual do Norte Fluminense Darcy Ribeiro, \\ 28013-603 Campos dos Goytacazes-RJ, Brazil
}

\begin{abstract}
Um novo diterpeno, $\left(5 S^{*}, 8 S^{*}, 9 R^{*}, 10 S^{*}\right)$-11 $\beta, 12 \beta$-epoxi-9 $\alpha$-hidróxi-19(4 $\left.\rightarrow 3\right)$ abeo-abieta3,13-dieno-19,18-olideo, e as substâncias conhecidas estemodina, D-manitol, ácido betulínico, uma mistura de $3 \beta-O-\beta$-D-glicopiranosil- $\beta$-sitosterol e $3 \beta-O$ - $\beta$-D-glicopiranosilestigmasterol, e 5,7,4'-triidróxi-3,8,3'-trimetoxiflavona, foram isolados das folhas e talos de Stemodia maritima. A elucidação estrutural de todas as substâncias baseou-se na interpretação de dados espectrais, principalmente RMN (1D e 2D) e espectrometria de massa (EM), envolvendo comparação com valores descritos na literatura.
\end{abstract}

A new diterpene, $\left(5 S^{*}, 8 S^{*}, 9 R^{*}, 10 S^{*}\right)-11 \beta, 12 \beta$-epoxy-9 $\alpha$-hydroxy-19(4 $\left.\rightarrow 3\right)$ abeo-abieta-3,13diene-19,18-olide, together with the known compounds stemodin, D-mannitol, betulinic acid, a mixture of $3 \beta-O-\beta$-D-glucopyranosyl- $\beta$-sitosterol and $3 \beta-O-\beta$-D-glucopyranosylstigmasterol and $5,7,4$ '-trihydroxy-3,8,3'-trimethoxyflavone were isolated from the leaves and stems of Stemodia maritima. Structural elucidation of all compounds was based on interpretation of spectral data, mainly NMR (1D and 2D) and MS, including comparison with values described in the literature.

Keywords: Stemodia maritima, Scrophulariaceae, diterpenes, steroids, flavonoids

\section{Introduction}

Stemodia Benth. is one of Scrophulariaceae genus and occurs in tropical and subtropical regions of the world. ${ }^{1}$ Although Stemodia comprises about 40 species, the chemical investigation of this genus is restricted to five species $^{4}$ from which flavonoids, ${ }^{2,3}$ labdane diterpenes ${ }^{4,5}$ and diterpenes derivatives with a rare tetracyclic skeletal, named stemodane, were isolated. This later class of diterpenes seems to be chemomarkers of Stemodia. ${ }^{6}$

S. maritima Linn. is a very common shrub that widely grows in Northeast Region of Brazil, near the sea coast, where it is known as "melosa". It has been used to treat stomachache, dropsy and swelling by local population,

*e-mail: angelamcarriaga@yahoo.com.br although toxic symptoms was reported in cattle. ${ }^{7}$ Stemodane diterpenes, including glycosides derivatives, possessing antiviral and cytotoxic properties were isolated from this species. ${ }^{6,8-10}$ The chemical composition and larvicidal activity of its essential oil were recently reported. ${ }^{11}$

On the course of the phytochemical investigation of S. maritima from the Northeast Region of Brazil, herein we report the non-volatile composition of this species. A new diterpene, $\left(5 S^{*}, 8 S^{*}, 9 R^{*}, 10 S^{*}\right)-11 \beta, 12 \beta$-epoxy$9 \alpha$-hydroxy-19(4 $\rightarrow 3)$ abeo-abieta-3,13-diene-19,18-olide (1), together with the known compounds stemodin (2) (Figure 1), D-mannitol, betulinic acid, a mixture of $3 \beta-O-\beta$-D-glucopyranosyl- $\beta$-sitosterol and $3 \beta-O-\beta-\mathrm{D}-$ glucopyranosylstigmasterol, and 5,7,4'-trihydroxy-3,8,3'trimethoxyflavone were isolated from the leaves and stems of this plant. Structural elucidation of all compounds was 


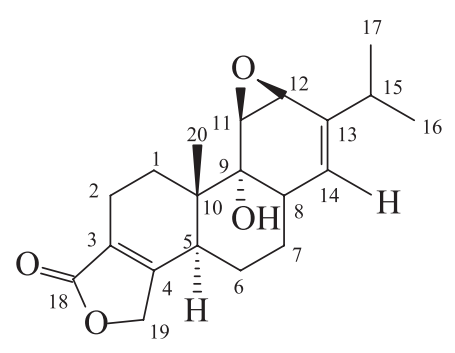

1

Figure 1. Compounds 1 e $\mathbf{2}$ isolated from Stemodia maritima.

based on the interpretation of spectral data, meanly NMR (1D and 2D) and MS, and comparison with literature data.

\section{Results and Discussion}

The molecular formula of compound $\mathbf{1}$ was established through HR-ESI-MS, which showed the quasi-molecular ion peak at $m / z 331.1799\left([\mathrm{M}+1]^{+}\right.$, corresponding to the molecular formula $\mathrm{C}_{20} \mathrm{H}_{26} \mathrm{O}_{4}$ and indicating eight degrees of unsaturation. EIMS from 1 showed the molecular ion peak at $\mathrm{m} / z, 330\left(\mathrm{C}_{20} \mathrm{H}_{26} \mathrm{O}_{4}, 5 \%\right)$ and additional peaks at $\mathrm{m} / \mathrm{z} 315\left(\mathrm{C}_{19} \mathrm{H}_{23} \mathrm{O}_{4}, 7 \%\right)$ and $\mathrm{m} / \mathrm{z} 287\left[\mathrm{C}_{17} \mathrm{H}_{19} \mathrm{O}_{4}, 100 \%\right]$, attributed to fragments $\mathbf{1 a}$ and $\mathbf{1 b}$, respectively (Figure 2). The presence of a hydroxyl absorption $\left(v_{\max } 3433 \mathrm{~cm}^{-1}\right)$ and an $\alpha, \beta$-unsatured- $\gamma$-lactone $\left(v_{\max } 1729 \mathrm{~cm}^{-1}\right)$ was inferred from its IR spectrum.

The ${ }^{1} \mathrm{H}$ NMR spectrum (Table 1 ) revealed the presence of an isopropyl group $\left(\delta_{\mathrm{H}} 1.03, \mathrm{~d}, J 6.8 \mathrm{~Hz}, 3 \mathrm{H}-16 ; \delta_{\mathrm{H}} 1.05\right.$, d, $J 6.8 \mathrm{~Hz}, 3 \mathrm{H}-17$; $\delta_{\mathrm{H}} 2.62$, sep, $\left.J 6.8 \mathrm{~Hz}, \mathrm{H}-15\right)$, a methyl group at $\delta_{\mathrm{H}} 1.01(3 \mathrm{H}, \mathrm{s}, 3 \mathrm{H}-20)$ attached to quaternary carbon, two oxygenated methine hydrogens at $\delta_{\mathrm{H}} 3.66(\mathrm{dd}$, $J 2.5$ and $1.9 \mathrm{~Hz}, \mathrm{H}-11)$ and $\delta_{\mathrm{H}} 4.40$ (brs, H-12), compatible with the presence of an epoxy ring, two deshielded hydrogen from a oxygenated methylene group at $\delta_{\mathrm{H}} 4.72$ (brdd, $J 17.2$ and $1.6 \mathrm{~Hz}, \mathrm{H}-19 \alpha$ ) and $\delta_{\mathrm{H}} 4.68$ (brdd, $J 17.2$ and $1.6 \mathrm{~Hz}$, $\mathrm{H}-19 \beta$ ), and an olefinic hydrogen at $\delta_{\mathrm{H}} 5.24$ (brd, J $5.0 \mathrm{~Hz}$, $\mathrm{H}-14)$.

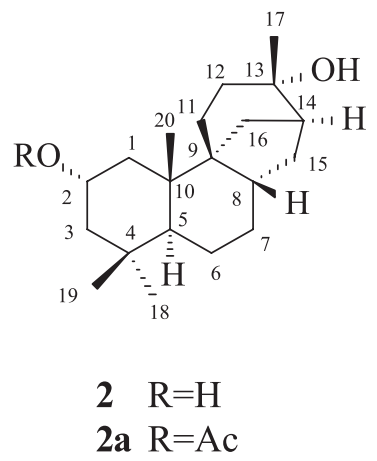

Analysis of BB and DEPT $135^{\circ}{ }^{13} \mathrm{C}$ NMR spectra (Table 1) revealed 20 lines, in accordance with the molecular formula $\mathrm{C}_{20} \mathrm{H}_{26} \mathrm{O}_{4}$. From these data it is possible to deduce the presence of the six non-protonated carbons: one carbonyl group $\left(\delta_{\mathrm{C}} 173.9\right)$, three $\mathrm{sp}^{2}$ carbons, one oxygenated $\mathrm{sp}^{3}$ carbon and one non-oxygenated $\mathrm{sp}^{3}$ carbon. Additionally, it was observed six methine carbons, including two $\mathrm{sp}^{3}$ oxygenated at $\delta_{\mathrm{C}} 66.6$ and 59.7 and one $\mathrm{sp}^{2}$ at $\delta_{\mathrm{C}} 121.8$; five methylene carbons, one of them oxygenated at $\delta_{\mathrm{C}} 70.4$, and three methyl carbons.

The aforementioned data were coherent with a non aromatic abietane-type diterpene that displays an epoxy ring, a tertiary hydroxyl group, an $\alpha, \beta$-unsaturated$\gamma$-lactone system and two double bonds, having some similarities with the diterpene triptolide. ${ }^{12}$

The location of these functions in the abietane skeleton was deduced through additional HMBC analysis (Table 1), which revealed the following long-range correlations: the epoxy hydrogens at $\delta_{\mathrm{H}} 3.66(\mathrm{H}-11)$ with $\mathrm{C}-13\left(\delta_{\mathrm{C}} 140.1\right.$, $\left.{ }^{3} J\right)$ and at $\delta_{\mathrm{H}} 4.4(\mathrm{H}-12)$ with $\mathrm{C}-13\left(\delta_{\mathrm{C}} 140.1,{ }^{2} J\right)$ and $\mathrm{C}-14\left(\delta_{\mathrm{C}} 121.8,{ }^{3} J\right)$; the isopropyl hydrogen at $\delta_{\mathrm{H}} 2.62$ $(\mathrm{H}-15$,$) with \mathrm{C}-13\left(\delta_{\mathrm{C}} 140.1,{ }^{2} J\right)$ and C-12 $\left(\delta_{\mathrm{C}} 66.6,{ }^{3} J\right)$; the olefin hydrogen at $\delta_{\mathrm{H}} 5.24$ with $\mathrm{C}-12\left(\delta_{\mathrm{C}} 66.6,{ }^{3} \mathrm{~J}\right)$, C-13 $\left(\delta_{\mathrm{C}} 140.1,{ }^{2} J\right)$ and $\mathrm{C}-15\left(\delta_{\mathrm{C}} 28.6,{ }^{3} J\right)$. The position of the hydroxyl group at C-9 was established based in the correlations of this oxymethine carbon $\left(\delta_{\mathrm{C}} 67.9\right)$ with the hydrogen of the methyl group $\left(3 \mathrm{H}-20, \delta_{\mathrm{H}} 1.01,{ }^{3} \mathrm{~J}\right)$, which

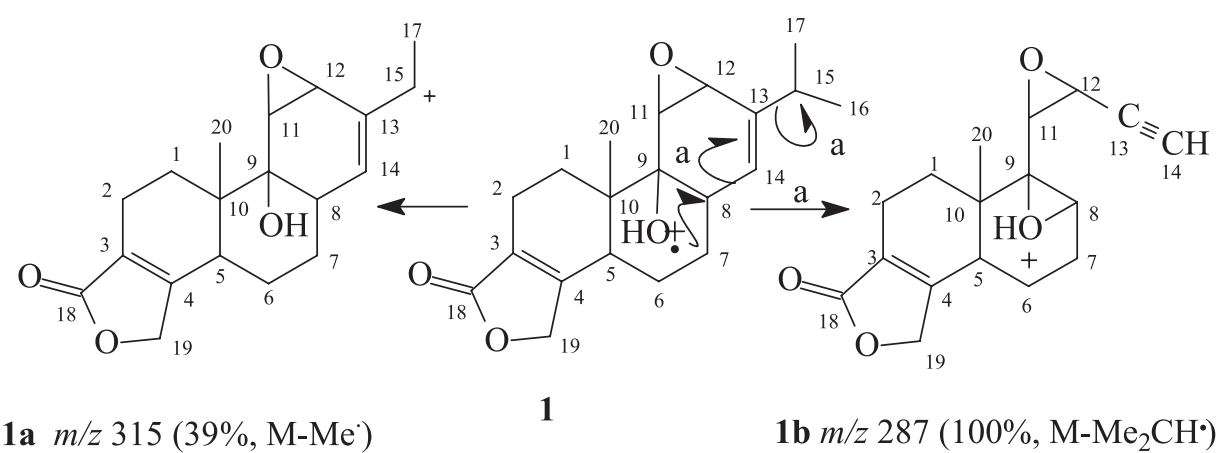

Figure 2. Fragments postulated to justify some of principal peaks observed in EIMS of $\mathbf{1}$. 
Table 1. ${ }^{1} \mathrm{H}$ and ${ }^{13} \mathrm{C}$ NMR data assignments for the compound $\mathbf{1}\left(\mathrm{CDCl}_{3}, 500 / 125 \mathrm{MHz}\right)$

\begin{tabular}{|c|c|c|c|c|c|c|}
\hline \multirow[b]{2}{*}{$\mathrm{C}$} & \multicolumn{2}{|c|}{ HSQC } & \multicolumn{2}{|c|}{ HMBC } & & \\
\hline & $\delta_{\mathrm{c}}$ & $\delta_{\mathrm{H}}$ & ${ }^{2} J_{\mathrm{CH}}$ & ${ }^{3} J_{\mathrm{CH}}$ & & \\
\hline 3 & 125.3 & - & $2 \mathrm{H}-2$ & H-1a, 2H-19 & & \\
\hline 4 & 162.0 & - & $2 \mathrm{H}-19$ & $2 \mathrm{H}-6$ & & \\
\hline 9 & 67.9 & - & H-8, H-11 & $\begin{array}{l}2 \mathrm{H}-1,2 \mathrm{H}-7 \\
\mathrm{H}-14,3 \mathrm{H}-20\end{array}$ & & \\
\hline 10 & 37.0 & - & $2 \mathrm{H}-1,3 \mathrm{H}-20$ & 2H-6, H-11 & & \\
\hline 13 & 140.1 & - & H-12, H-14, H-15 & $\begin{array}{c}\mathrm{H}-8, \mathrm{H}-11,3 \mathrm{H}-16, \\
3 \mathrm{H}-17\end{array}$ & & \\
\hline 18 & 173.9 & - & - & $2 \mathrm{H}-19$ & \multicolumn{2}{|c|}{${ }^{1} \mathrm{H}-{ }^{-} \mathrm{H}-\mathrm{NOESY}$} \\
\hline $\mathrm{CH}$ & & & & & $\mathrm{H}$ & nOe \\
\hline 5 & 44.2 & $2.51(\mathrm{~m})$ & $2 \mathrm{H}-6$ & $2 \mathrm{H}-1,2 \mathrm{H}-7,3 \mathrm{H}-20$ & H-5 $\alpha$ & $\begin{array}{l}\mathrm{H}-1 \alpha, \mathrm{H}-6 \alpha \\
\mathrm{H}-7 \alpha, \mathrm{H}-19 \alpha\end{array}$ \\
\hline 8 & 34.6 & $2.86(\mathrm{dd}, 12.2,5.0)$ & $2 \mathrm{H}-7$ & $2 \mathrm{H}-6, \mathrm{H}-14$ & $\mathrm{H}-8 \beta$ & H- $6 \beta, \mathrm{H}-7 \beta, 3 \mathrm{H}-20 \beta$ \\
\hline 11 & 59.7 & $3.66(\mathrm{dd}, 2.5,1.9)$ & & $\mathrm{H}-8$ & $\mathrm{H}-11 \alpha$ & $2 \mathrm{H}-1$ \\
\hline 12 & 66.6 & 4.40 (brs) & $\mathrm{H}-11$ & H-14, H-15 & $H-12 \alpha$ & $\mathrm{H}-15,3 \mathrm{H}-26,3 \mathrm{H}-27$ \\
\hline 14 & 121.8 & 5.24 (brd, 5.0) & $\mathrm{H}-8$ & $2 \mathrm{H}-7, \mathrm{H}-12,2 \mathrm{H}-7$ & $\mathrm{H}-14$ & $\begin{array}{c}2 \mathrm{H}-7, \mathrm{H}-8 \beta, \mathrm{H}-15 \\
3 \mathrm{H}-26,3 \mathrm{H}-27\end{array}$ \\
\hline 15 & 28.6 & $2.62(\mathrm{sep}, 6.8)$ & $3 \mathrm{H}-16,3 \mathrm{H}-17$ & $\mathrm{H}-14$ & - & - \\
\hline $\mathrm{CH}_{2}$ & & & & & - & - \\
\hline 1 & 28.4 & $\begin{array}{c}\alpha 1.77(\mathrm{dd}, 12.8,5.3) \\
\beta 1.36(\mathrm{~m})\end{array}$ & - & $3 \mathrm{H}-20$ & - & - \\
\hline 2 & 17.7 & $\begin{array}{l}2.38(\mathrm{~m}) \\
2.20(\mathrm{~m})\end{array}$ & $2 \mathrm{H}-1$ & - & - & - \\
\hline 6 & 22.7 & $\begin{array}{l}\alpha 1.67(\mathrm{~m}) \\
\beta 1.62(\mathrm{~m})\end{array}$ & $2 \mathrm{H}-7$ & - & - & - \\
\hline 7 & 32.9 & $\begin{array}{l}\beta 2.11(\mathrm{~m}) \\
\alpha 1.07(\mathrm{~m})\end{array}$ & $2 \mathrm{H}-6, \mathrm{H}-8$ & - & - & - \\
\hline 19 & 70.4 & $\begin{array}{l}4.72 \text { (brdd, } 17.2,1.6) \\
4.68 \text { (brdd, 17.2, 1.6) }\end{array}$ & - & - & - & - \\
\hline $\mathrm{CH}_{3}$ & & & & & - & - \\
\hline 16 & 22.9 & $1.03(\mathrm{~d}, 6.8)$ & $\mathrm{H}-15$ & $3 \mathrm{H}-17$ & - & - \\
\hline 17 & 20.9 & $1.05(\mathrm{~d}, 6.8)$ & $\mathrm{H}-15$ & $3 \mathrm{H}-16$ & - & - \\
\hline 20 & 14.0 & $1.01(\mathrm{~s})$ & & $2 \mathrm{H}-1$ & $3 \mathrm{H}-20$ & $\begin{array}{c}\mathrm{H}-1 \beta, \mathrm{H}-2 \beta, \mathrm{H}-6 \beta, \\
\mathrm{H}-8 \beta\end{array}$ \\
\hline
\end{tabular}

is generally present in abietane-type diterpenoids. ${ }^{13}$ Finally, the butenolide ring involving the carbons C-3, C-4, C-18 and $\mathrm{C}-19$ was located by the correlations of the methylene hydrogens at $\delta_{\mathrm{H}} 4.72$ and $4.68(2 \mathrm{H}-19)$ with C-4 $\left(\delta_{\mathrm{C}} 162.0\right.$, $\left.{ }^{2} J\right), \mathrm{C}-3\left(\delta_{\mathrm{C}} 125.3,{ }^{3} \mathrm{~J}\right)$ and C-18 $\left(\delta_{\mathrm{C}} 173.9,{ }^{3} \mathrm{~J}\right)$.

The relative configuration of $\mathbf{1}$ (Figure 3 ) was assigned by the analysis of the ${ }^{1} \mathrm{H}-{ }^{1} \mathrm{H}-\mathrm{NOESY}$ spectrum. The $\beta$-orientation of the epoxy function (11,12 $\beta$-epoxide) was determined by the dipolar interactions of the hydrogen at $\delta_{\mathrm{H}} 3.66(\mathrm{H}-11)$ with $2 \mathrm{H}-1\left(\delta_{\mathrm{H}} 1.77\right.$ and 1.36$)$. In addition, the methyl signal at $\delta_{\mathrm{H}} 1.01(3 \mathrm{H}-20)$ exhibited cross-peaks with the hydrogens at $\delta_{\mathrm{H}} 2.86(\mathrm{H}-8), \delta_{\mathrm{H}} 2.20(\mathrm{H}-2 \beta)$ and $\delta_{\mathrm{H}} 1.62(\mathrm{H}-6 \beta)$. The hydrogen at $\delta_{\mathrm{H}} 2.51(\mathrm{H}-5)$ showed dipolar interaction with the hydrogens at $\delta_{\mathrm{H}} 1.77(\mathrm{H}-1 \alpha)$, $1.67(\mathrm{H}-6 \alpha)$ and $1.07(\mathrm{H}-7 \alpha)$. Based on these correlations, the hydroxyl group at C-9 was established at $\alpha$ position (Figure 3). Therefore, all these data allowed to establish the structure of 1 as $\left(5 S^{*}, 8 S^{*}, 9 R^{*}, 10 S^{*}\right)-11 \beta, 12 \beta$-epoxy$9 \alpha$-hydroxy-19(4 $\rightarrow 3)$ abeo-abieta-3,13-diene-19,18-olide.

Compound 2 was obtained as colorless crystal and its molecular formula $\mathrm{C}_{20} \mathrm{H}_{34} \mathrm{O}_{2}$ was deduced by EIMS ([M] $]^{+}$, $\mathrm{m} / \mathrm{z}, 306)$ and ${ }^{1} \mathrm{H}$ and ${ }^{13} \mathrm{C}$ NMR analysis. Its IR spectrum showed hydroxyl absorption at $v_{\max } 3311 \mathrm{~cm}^{-1}$. All spectral data were in accordance with the structure of the stemodin (2), a stemodane-type diterpene previously isolated from Stemodia species. ${ }^{6,8}$ 

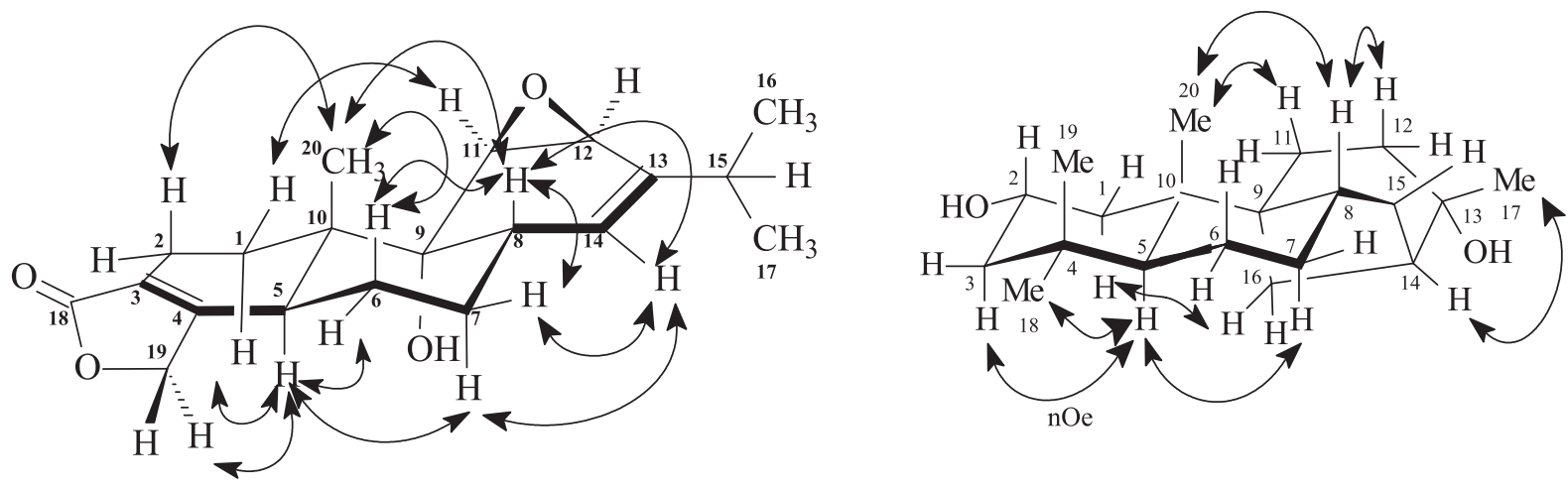

Figure 3. Selected NOESY correlations (depicted by double arrows) for compounds $\mathbf{1}$ e $\mathbf{2}$.

Compound 2 was submitted to acetylation with pyridine and acetic anhydride (see Experimental section), yielding $\mathbf{2} \mathbf{a}^{8}$ (Figure 1). The 1D and 2D NMR spectral data of $\mathbf{2}$ and of its acetyl derivative (2a) were also used to complete ${ }^{1} \mathrm{H}$ and ${ }^{13} \mathrm{C}$ chemical shifts described in Table 2. Dipolar interactions observed from ${ }^{1} \mathrm{H}-{ }^{1} \mathrm{H}-\mathrm{NOESY}$ analysis of $\mathbf{2}$ are summarized in Figure 3.

The other isolated compounds were identified on the basis of their spectral analysis and comparison with the literature data.

Table 2. ${ }^{1} \mathrm{H}$ and ${ }^{13} \mathrm{C}$ NMR data assignments for the compounds $\mathbf{2}$ and $\mathbf{2 a}\left(\mathrm{CDCl}_{3}, 500 / 125 \mathrm{MHz}\right)$

\begin{tabular}{|c|c|c|c|c|c|c|}
\hline \multirow[b]{2}{*}{$\mathrm{C}$} & \multicolumn{4}{|c|}{$2 a$} & \multicolumn{2}{|c|}{2} \\
\hline & $\delta_{\mathrm{C}}$ & $\delta_{\mathrm{H}}$ & ${ }^{2} J_{\mathrm{CH}}$ & ${ }^{3} J_{\mathrm{CH}}$ & $\delta_{\mathrm{C}}$ & $\delta_{\mathrm{H}}$ \\
\hline 4 & 34.9 & - & $3 \mathrm{H}-18,3 \mathrm{H}-19$ & - & 35.0 & - \\
\hline 9 & 50.3 & - & $2 \mathrm{H}-11,2 \mathrm{H}-16,3 \mathrm{H}-20$ & $\mathrm{H}-1 \mathrm{~b}, \mathrm{H}-14$ & 50.3 & - \\
\hline 10 & 40.3 & - & $2 \mathrm{H}-1,3 \mathrm{H}-20$ & H-6a & 40.4 & - \\
\hline 13 & 72.6 & - & $\mathrm{H}-12 \mathrm{~b}, \mathrm{H}-14,3 \mathrm{H} 20$ & $2 \mathrm{H}-15,2 \mathrm{H}-16$ & 72.6 & - \\
\hline $\mathrm{AcO}$ & 170.8 & - & - & $\mathrm{H}-2$ & - & - \\
\hline \multicolumn{7}{|l|}{$\mathrm{CH}$} \\
\hline 2 & 69.4 & $4.91(\mathrm{tt}, 11.8,3.8)$ & $2 \mathrm{H}-1,2 \mathrm{H}-3$ & $3 \mathrm{H}-18,3 \mathrm{H}-19$ & 65.5 & $3.77(\mathrm{tt}, 11.9,3.7)$ \\
\hline 5 & 46.8 & 1.24 & - & $2 \mathrm{H}-3,3 \mathrm{H}-18,3 \mathrm{H}-19$ & 46.7 & 1.24 \\
\hline 8 & 37.0 & 1.72 & - & $2 \mathrm{H}-16$ & 37.0 & 1.76 \\
\hline 14 & 46.3 & 1.97 & $2 \mathrm{H}-15,2 \mathrm{H}-16$ & $3 \mathrm{H}-17$ & 46.3 & 1.96 \\
\hline \multicolumn{7}{|l|}{$\mathrm{CH}_{2}$} \\
\hline 1 & 41.9 & $2.00,1.28$ & $\mathrm{H}-2$ & $2 \mathrm{H}-3$ & 46.0 & $1.99,1.21$ \\
\hline 3 & 46.7 & $1.72,1.08$ & $\mathrm{H}-2$ & $2 \mathrm{H}-1, \mathrm{H}-5$ & 50.9 & $1.78,1.09(\mathrm{t}, 11.9)$ \\
\hline 6 & 22.1 & $1.40,1.18$ & $2 \mathrm{H}-7$ & & 22.2 & $1.42,1.21$ \\
\hline 7 & 36.5 & $1.92,1.72$ & $2 \mathrm{H}-6, \mathrm{H}-8$ & & 36.6 & $1.92,1.15$ \\
\hline 11 & 27.9 & $1.57,1.40$ & $\mathrm{H}-12 \mathrm{a}$ & H-16a & 27.9 & $1.65,1.40$ \\
\hline 12 & 33.0 & $1.52,1.32$ & H-11a & H-14, 3H-17 & 33.0 & $1.57,1.43$ \\
\hline 15 & 38.2 & $1.70,1.25$ & H-14, 2H-15 & & 38.3 & $1.74,1.26$ \\
\hline 16 & 30.2 & $1.80(\mathrm{~d}, 11.9), 1.70$ & - & $2 \mathrm{H}-11, \mathrm{H}-15 \mathrm{a}$ & 30.2 & 1.82 (brd, 11.6), 1.74 \\
\hline \multicolumn{7}{|l|}{$\mathrm{CH}_{3}$} \\
\hline 17 & 28.3 & $1.12(\mathrm{~s})$ & - & $\mathrm{H}-14$ & 28.3 & $1.13(\mathrm{~s})$ \\
\hline 18 & 34.7 & $0.96(\mathrm{~s})$ & - & $2 \mathrm{H}-3, \mathrm{H}-5,3 \mathrm{H}-19$ & 34.8 & $0.96(\mathrm{~s})$ \\
\hline 19 & 23.7 & $0.95(\mathrm{~s})$ & - & $2 \mathrm{H}-3, \mathrm{H}-5,3 \mathrm{H}-18$ & 23.9 & $0.93(\mathrm{~s})$ \\
\hline 20 & 19.5 & $1.05(\mathrm{~s})$ & - & $2 \mathrm{H}-1$ & 19.8 & $0.99(\mathrm{~s})$ \\
\hline $\mathrm{AcO}$ & 21.7 & $2.02(\mathrm{~s})$ & - & - & - & - \\
\hline
\end{tabular}




\section{Experimental}

\section{General experimental procedures}

Melting points were obtained from a Mettler FP82HT apparatus and are uncorrected. IR spectra were recorded using a Perkin Elmer 1000 FT-IR spectrophotometer. Optical rotations were measured on a Perkin Elmer 341 polarimeter. High resolution electrospray ionization mass spectra (ESI-MS/MS), in positive mode, was performed on a QTOF Micromass spectrometer (QqTOF, MicromassUK). ${ }^{1} \mathrm{H}$ and ${ }^{13} \mathrm{C}$ NMR spectra were recorded on a Bruker Avance DRX-500 (500 MHz for ${ }^{1} \mathrm{H}$ and $125 \mathrm{MHz}$ for ${ }^{13} \mathrm{C}$ ); chemical shifts are given in ppm relative to residual $\mathrm{CHCl}_{3}$ (7.27 and 77.23 ppm). Silica Gel 60 (Merck, 230-400 mesh) was used for analytical TLC. Silica gel 60 (Merck, $60 \mathrm{~F}_{254}, 0.2 \mathrm{~mm}$ ) was used for column chromatography. All compounds were visualized on TLC by spraying with vanillin/perchloric acid/EtOH followed by heating.

\section{Plant material}

S. maritima was collected during the flowering stage in September 2006 along the Flexeiras Beach, Ceara Cost, Northeast of Brazil. The plant was identified by Dr. F. S. Cavalcanti and Prof. E. P. Nunes from the Herbário Prisco Bezerra (EAC), Universidade Federal do Ceará, Fortaleza, Brazil, where a voucher specimen (\# 38483) is deposited.

\section{Extraction and isolation}

The fresh stems (200.0 g) of S. maritima were exhaustively extracted with ethanol, at room temperature, to obtain a crude material, composed by a precipitate, which was recrystalized from methanol to give D-mannitol ${ }^{14}$ (80.0 mg, 0.04\%).

The aqueous extract obtained after the essential oil extraction (hydrodistillation) of the fresh stems of $S$. maritima was submitted to liquid-liquid partition with hexane/MeOH (3:7). The hexane fraction (340.0 g) was submitted to column chromatography on silica gel column, using a gradient solvent system of hexane and $\mathrm{CH}_{2} \mathrm{Cl}_{2}$. Chromatography of the subfraction hexane (380.0 mg) using hexane/EtOAc mixtures with increasing polarity yielded betulinic acid ${ }^{15}(8.5 \mathrm{mg}, 0.0025 \%)$. Successive flash chromatography of $\mathrm{CH}_{2} \mathrm{Cl}_{2}$ subfraction (2.0 g) using 0-100\% $\mathrm{CH}_{2} \mathrm{Cl}_{2} /$ EtOAc provided a mixture of $3 \beta-O-\beta$-D-glucopyranosyl- $\beta$-sitosterol and $3 \beta-O-\beta$-Dglucopyranosylstigmasterol $^{16}(8.2 \mathrm{mg}, 0.0024 \%)$.

After extraction of the essential oils from the leaves of S. maritime by hydrodistillation, the aqueous extract was subjected to liquid-liquid partition with ethyl acetate. The organic fraction $(4.0 \mathrm{~g})$ was chromatographed over silica gel with $\mathrm{CHCl}_{3}$, EtOAc and $\mathrm{MeOH}$ to afford three subfractions F1-F3. Successive flash column chromatography of F1 (1.2 g), previously eluted from $\mathrm{CHCl}_{3}$, yielded $2(45.3 \mathrm{mg}$, $1.13 \%$ ) after elution with $\mathrm{CHCl}_{3} /$ hexane $7: 3$. From these same column, fraction $\mathrm{CHCl}_{3} /$ hexane 9:1 (180.0 mg) was also obtained and rechromatographed over silica gel using the same eluent system to afforded 5,7,4'-trihydroxy-3,8,3'trimethoxyflavone ${ }^{17}(6.5 \mathrm{mg}, 0.0019 \%)$ and $1(15.6 \mathrm{mg}$, $0.39 \%)$.

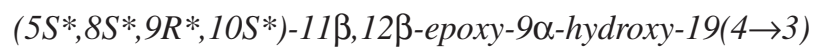
abeo-abieta-3,13-diene-19,18-olide (1)

Crystalline Solid; mp 264.6-266.5 ${ }^{\circ} \mathrm{C}$; IR (film, KBr) $v_{\max } / \mathrm{cm}^{-1}: 3433,2962,2866,1729,1663,1453,1344$, 1036; HREIMS, $m / z$ 331.1799, required $m / z$ 331.1909; $[\alpha]_{\mathrm{D}}^{25}=-12.9^{\circ}\left(c 1.0, \mathrm{CHCl}_{3}\right)$.

\section{Stemodin (2)}

Crystalline Solid; mp 189.9-192.4 ${ }^{\circ} \mathrm{C}$; IR (film, KBr) $v_{\max } / \mathrm{cm}^{-1}: 3311,2954,1463,1367,1217,1032$; EIMS, $m / z$ $306\left(\mathrm{M}^{+\cdot}\right), 291,288,273,232,217,161,94$.

The structures of known compounds were established by $1 \mathrm{D}{ }^{1} \mathrm{H}$ and ${ }^{13} \mathrm{C}\left(\left\{{ }^{1} \mathrm{H}\right\}\right.$ and DEPT $)$ and $2 \mathrm{D}{ }^{1} \mathrm{H}-{ }^{1} \mathrm{H}-\mathrm{COSY}$, HSQC and HMBC NMR spectral data (Table 2) and by comparison of their spectroscopy data with those reported in the literature. ${ }^{6}$

\section{Acetylation of $\mathbf{2}$}

To a solution of compound $2(24.0 \mathrm{mg})$ in pyridine $(0.5 \mathrm{~mL})$ were added $\mathrm{Ac}_{2} \mathrm{O}(1.0 \mathrm{~mL})$ and catalytic amount of DMAP. The mixture was stirred for $5 \mathrm{~h}$ at room temperature. Subsequent workup afforded a residue that was chromatographed using hexane/ $\mathrm{CHCl}_{3}(1: 1)$, hexane/ $\mathrm{CHCl}_{3}$ (1:3) as eluent to yield compound $\mathbf{2} \mathbf{a}^{8}$ (12.0 $\mathrm{mg}$, $50.0 \%$ ) as a colorless solid.

\section{Supplementary Information}

Supplementary data are available free of charge at http://jbcs.sbq.org.br, as PDF file.

\section{Acknowledgments}

The authors acknowledge the financial support provided by Brazilian Agencies CNPq, CAPES, FUNCAP and PRONEX, and are grateful to Fundação de Amparo a Pesquisa do Estado do Rio de Janeiro (FAPERJ-RJ-Brazil) for the fellowship to visitant research and grants and to the Centro Nordestino de Aplicação e Uso da Ressonância 
Magnética Nuclear (CENAUREMN), Universidade Federal do Ceará (UFC), Fortaleza-CE, for the support to perform of the NMR (1D and 2D) spectra and to the Laboratório Thomson de Espectrometria de Massas, IQ-Unicamp, Campinas-SP, Brazil, for the high resolution mass spectra.

\section{References}

1. Chamy, M. C.; Piovano, M.; Garbarino, J. A.; Gambaro, V.; Phytochemistry 1991, 30, 1719.

2. Ramesh, P.; Nair, A. G. R.; Subramanian, S. S.; Curr. Sci. 1979, $48,67$.

3. Rodrigues, F. E. A.; MSc Dissertation, Universidade Federal do Ceará, Brazil, 2008.

4. Da Silva, W. M. B.; Assunção, J. C. da C.; Araújo, R. M.; Silveira, E. R.; Pessoa, O. D. L.; J. Braz. Chem. Soc. 2009, 20, 37.

5. Da Silva, L. L. D.; Nascimento, M. S.; Cavalheiro, A. J.; Silveira, D. H. S.; Castro-Gamboa, I.; Furlan, M.; Bolzani, V. S.; J. Nat. Prod. 2008, 71, 1291.

6. Hufford, C. D.; Oguntimein, B. O.; Muhammad, I.; J. Nat. Prod. 1992, 55, 48 .
7. Silva, D. M.; Correa, F. R.; Medeiros, R. M. T.; Oliveira, O. F.; Pesquisa Vet. Brasil. 2006, 26, 223.

8. Manchand, P. S.; White, J. D.; Wright, H.; Clardy, J.; J. Am. Chem. Soc. 1973, 95, 2705.

9. Martin, G. D. A.; Reynolds, W. F.; Reese, P. B.; Phytochemistry 2005, 66, 901.

10. Hufford, C. D.; Badria, F. A.; Abau-Karam, M.; Shier, W. T.; Rogers, R. D.; J. Nat. Prod. 1991, 54, 1543.

11. Arriaga, A. M. C.; Rodrigues, F. E. A.; Lemos, T. L. G.; de Oliveira, M. C. F.; Lima, J. Q.; Santiago, G. M. P.; Braz-Filho, R.; Mafezoli, J.; Nat. Prod. Commun. 2007, 2, 1237.

12. Ghisalberti, E. L.; Fitoterapia 1997, 68, 303.

13. Duan, H.; Kawazoe, K.; Bando, M.; Kido, M.; Takaishi, Y.; Phytochemistry 1997, 46, 535.

14. Pouchert, C. J.; Behnke, J.; The Aldrich Library of ${ }^{13} \mathrm{C}$ and ${ }^{1} \mathrm{H}$ FT NMR Spectra, Aldrich Chemical Company: Milwakee, 1993.

15. Kolak, U.; Turk. J. Chem. 2007, 31, 363.

16. Costa, H. N. R.; Santos, M. C.; Alcântara, A. F. C.; Silva, M. C.; França, R. C.; Piló-Veloso, D.; Quim. Nova 2008, 31, 744. 17. Roitman, J. N.; James, L. F.; Phytochemistry 1985, 24, 835.

Received: June 24, 2009 Web Release Date: March 25, 2010 


\section{Diterpene and other Constituents from Stemodia maritima (Scrophulariaceae)}

\section{Francisco E. A. Rodrigues, ${ }^{a}$ Jefferson Q. Lima, ${ }^{a, b}$ Maria da Conceição F. de Oliveira, ${ }^{a}$} Jackson N. Vasconcelos, ${ }^{a}$ Gilvandete M. P. Santiago, ${ }^{a, c}$ Jair Mafezoli, ${ }^{a}$ Raimundo Braz-Filho ${ }^{d}$ and Angela M. C. Arriaga ${ }^{*, a}$

${ }^{a}$ Curso de Pós-Graduação em Química, Departamento de Química Orgânica e Inorgânica, Universidade Federal do Ceará, CP 6036, 60451-970 Fortaleza-CE, Brazil

${ }^{b}$ Curso de Engenharia Ambiental, Instituto Federal do Ceará, Campus Juazeiro do Norte, 63040-000 Juazeiro do Norte-CE, Brazil

'Departamento de Farmácia, Universidade Federal do Ceará, 60430-370 Fortaleza-CE, Brazil

${ }^{d}$ Centro de Ciências e Tecnologias, Universidade Estadual do Norte Fluminense Darcy Ribeiro, 28013-603 Campos dos Goytacazes-RJ, Brazil

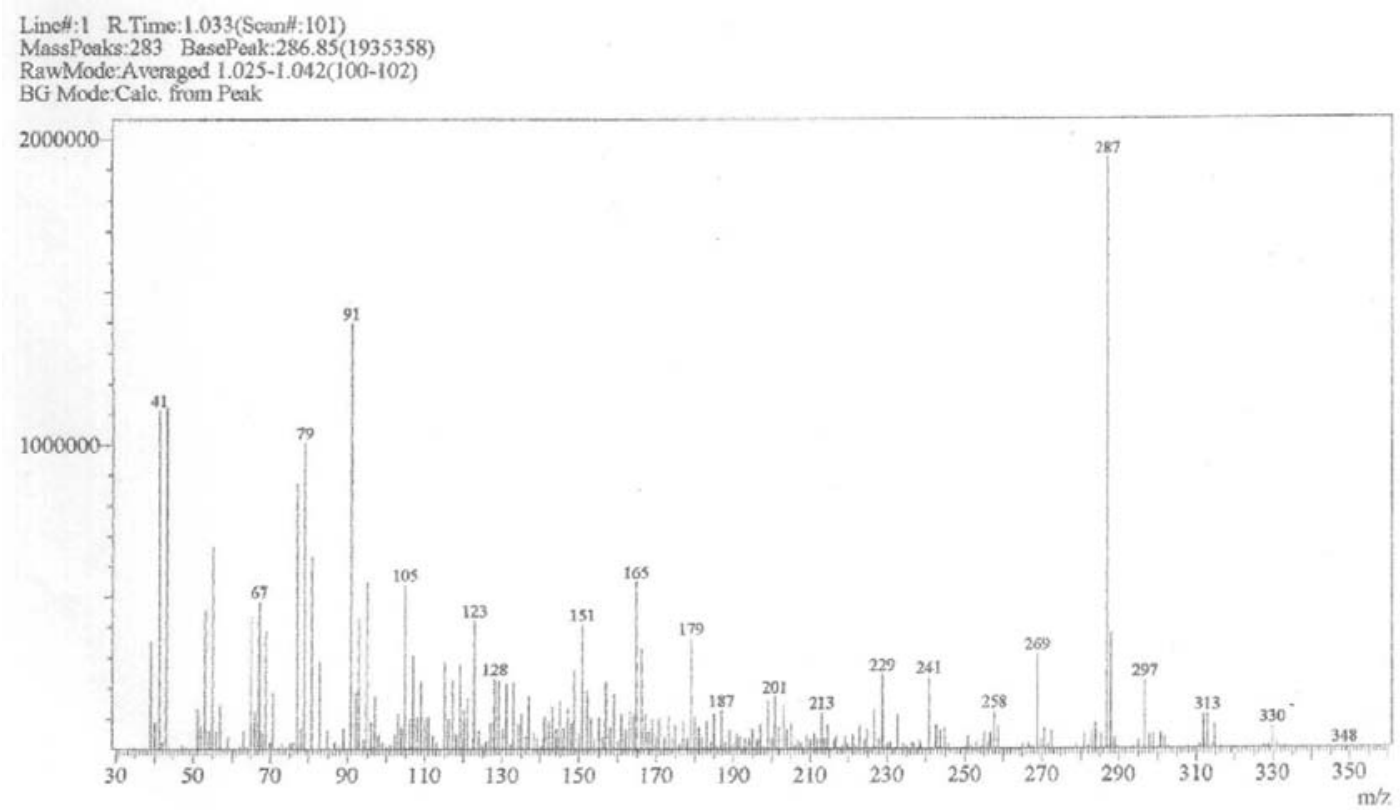

Figure S1a. EI-MS of compound (1) isolated from leaves of Stemodia maritima. 

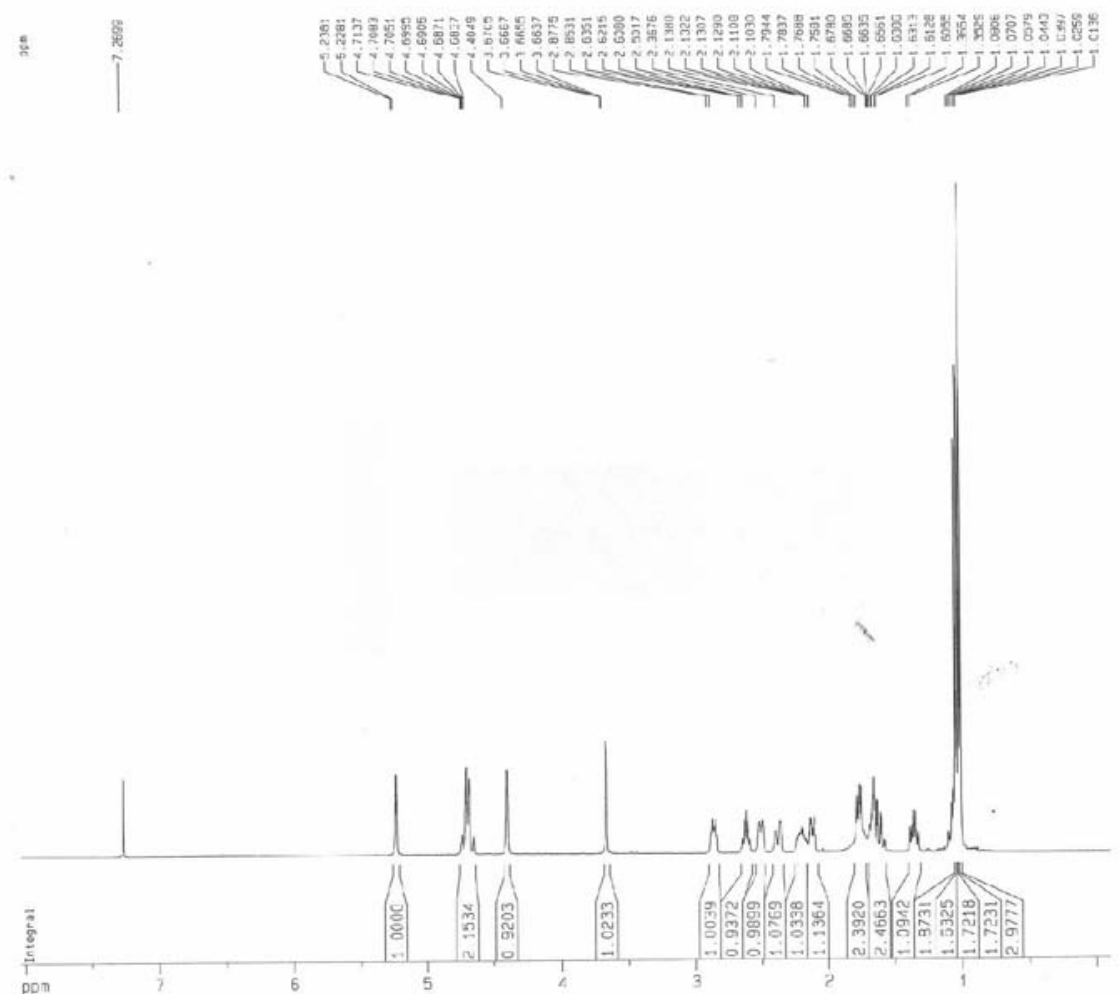

Figure S1b. ${ }^{1} \mathrm{H}$ NMR spectrum $\left(\mathrm{CDCl}_{3}, 500 \mathrm{MHz}\right)$ of compound (1) isolated from leaves of Stemodia maritima.
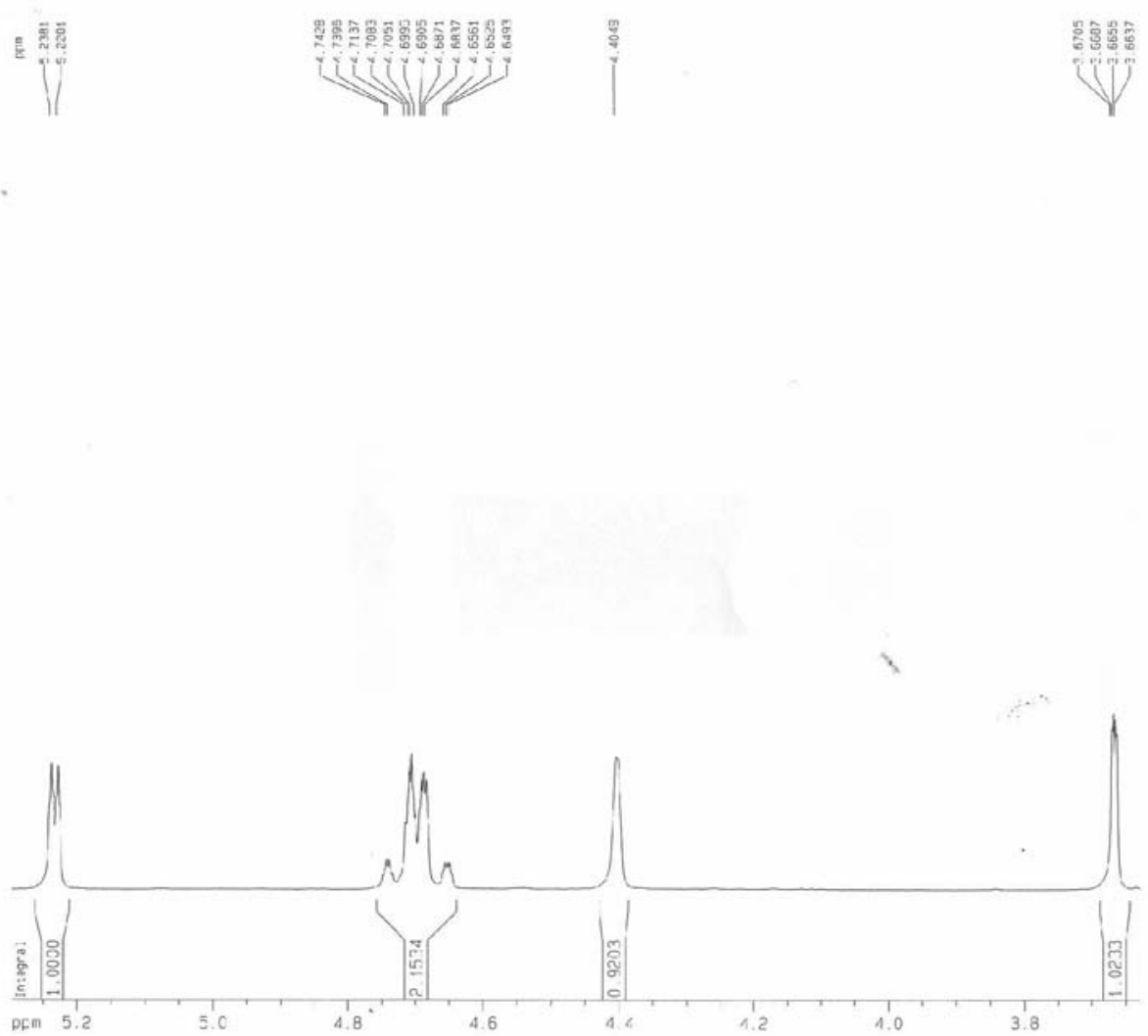

Figure S2. Expansion $1{ }^{1} \mathrm{H}$ NMR spectrum $\left(\mathrm{CDCl}_{3}, 500 \mathrm{MHz}\right)$ of compound (1) isolated from leaves of Stemodia maritima. 

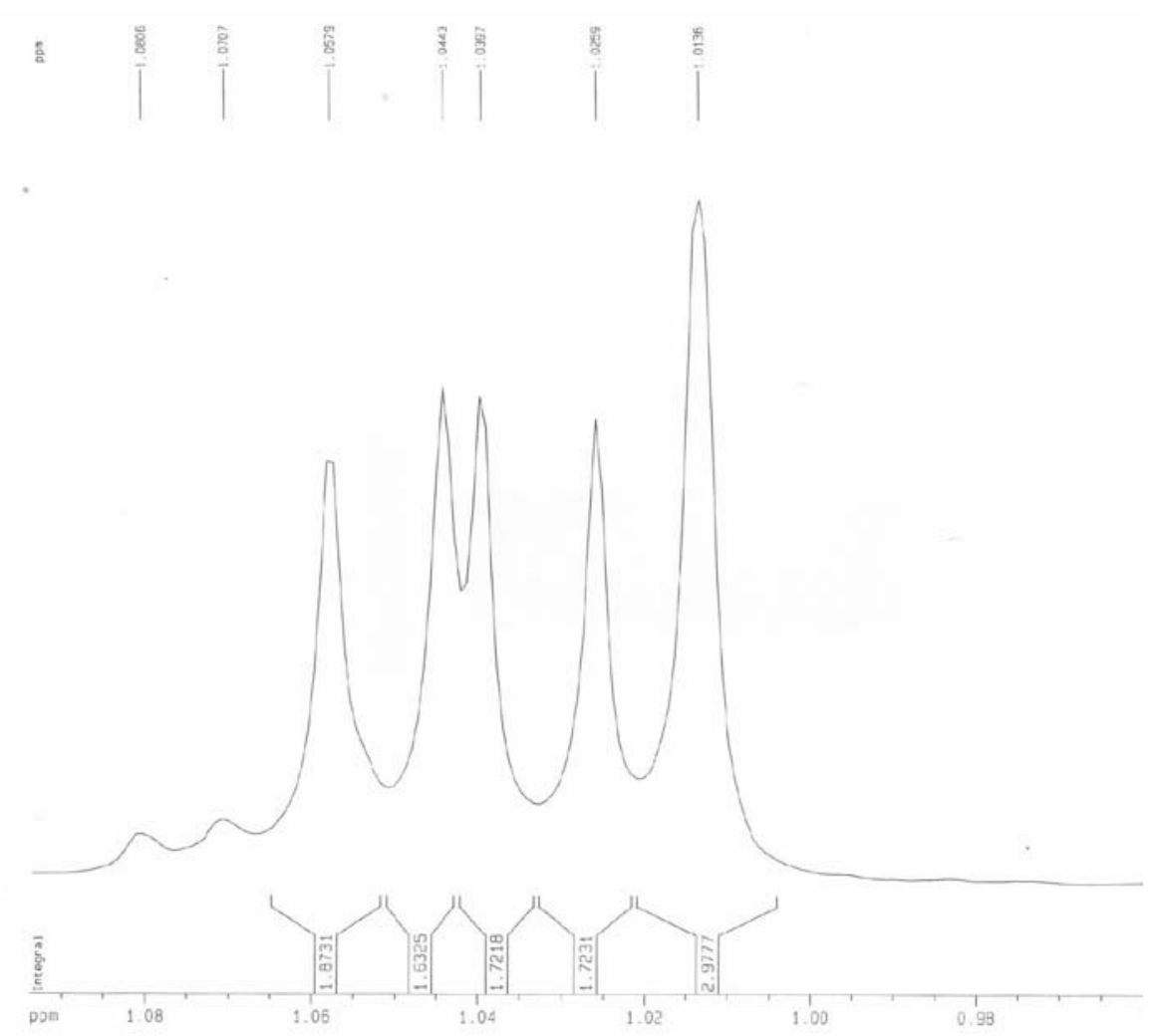

Figure S3. Expansion $2{ }^{1} \mathrm{H}$ NMR spectrum $\left(\mathrm{CDCl}_{3}, 500 \mathrm{MHz}\right)$ of compound (1) isolated from leaves of Stemodia maritima.

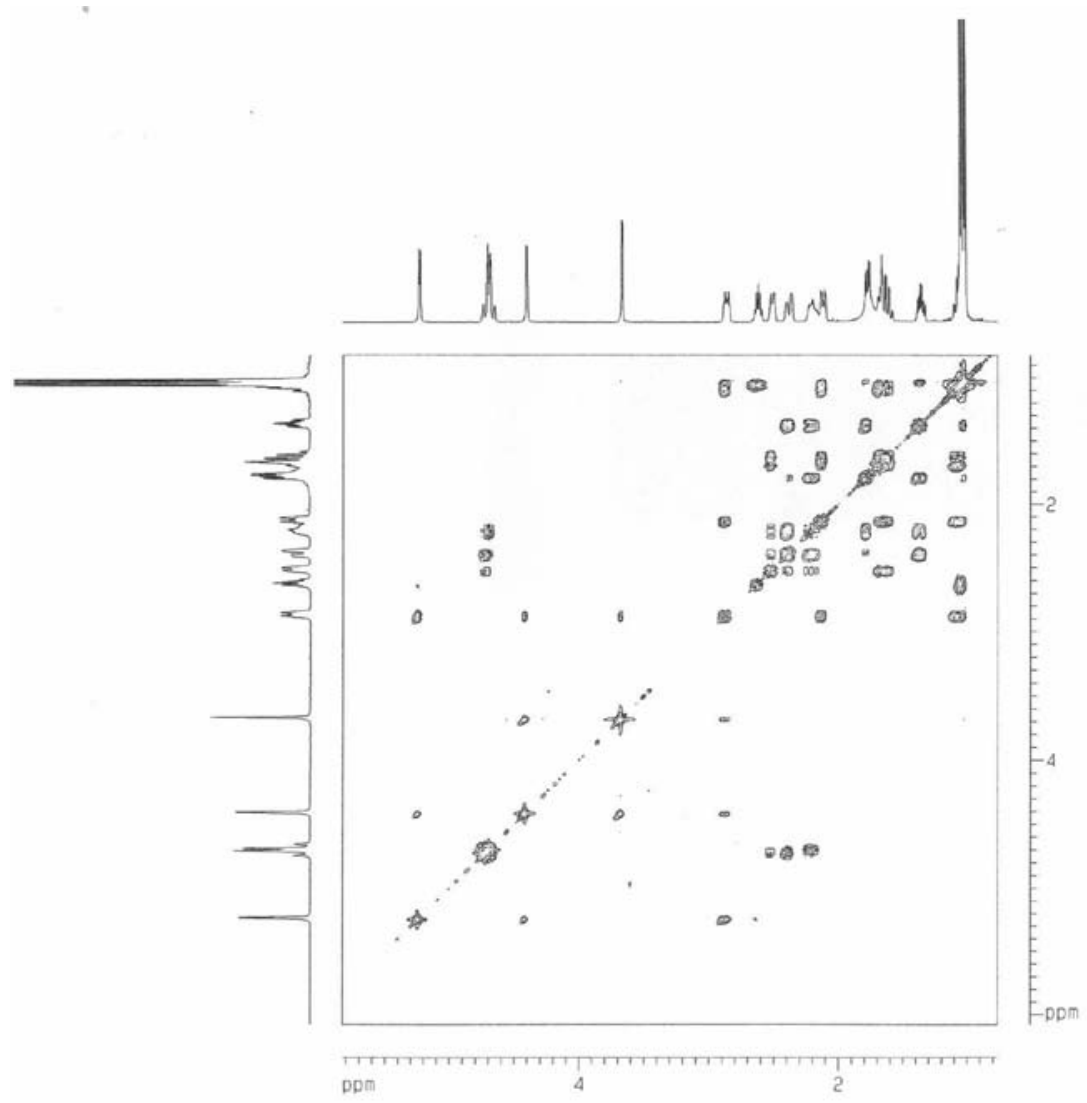

Figure S4. COSY NMR experiment $\left(\mathrm{CDCl}_{3}, 500 \mathrm{MHz}\right)$ of compound (1) isolated from leaves of Stemodia maritima. 


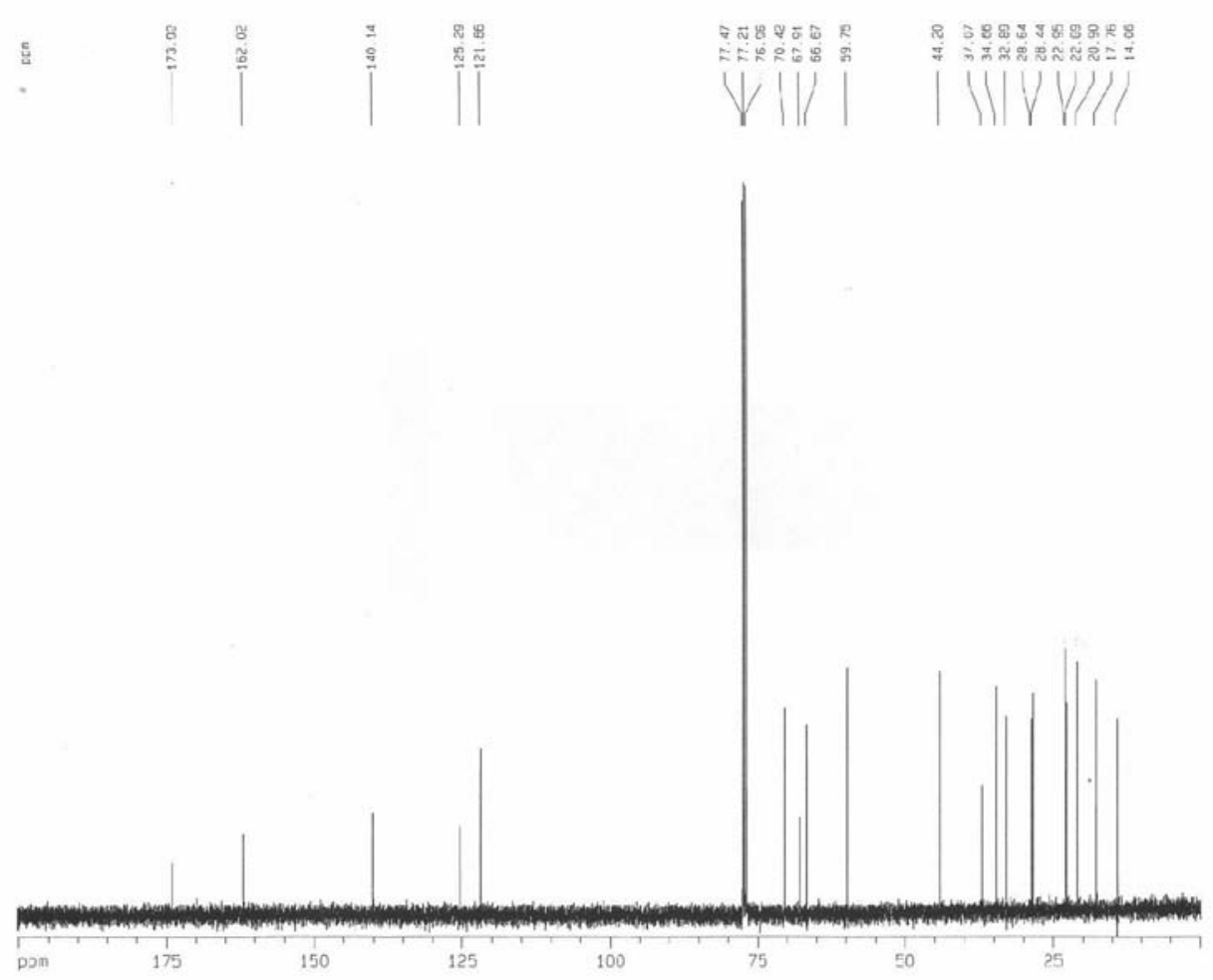

Figure S5. ${ }^{13} \mathrm{C}$ RMN spectrum $\left(\mathrm{CDCl}_{3}, 125 \mathrm{MHz}\right)$ of compound (1) isolated from leaves of Stemodia maritima

玄
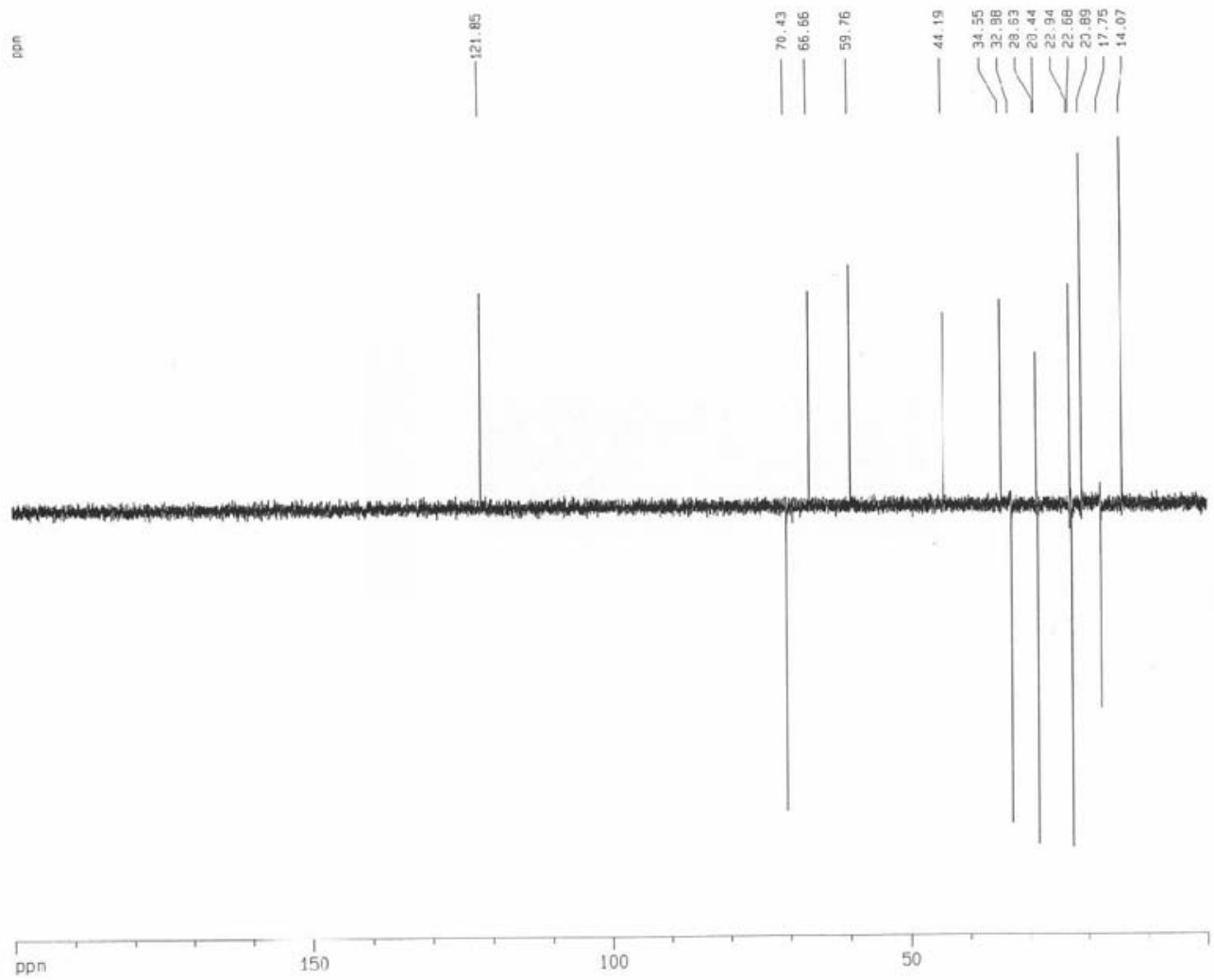

Figure S6. DEPT NMR experiment $\left(\mathrm{CDCl}_{3}, 125 \mathrm{MHz}\right)$ of compound (1) isolated from leaves of Stemodia maritima. 


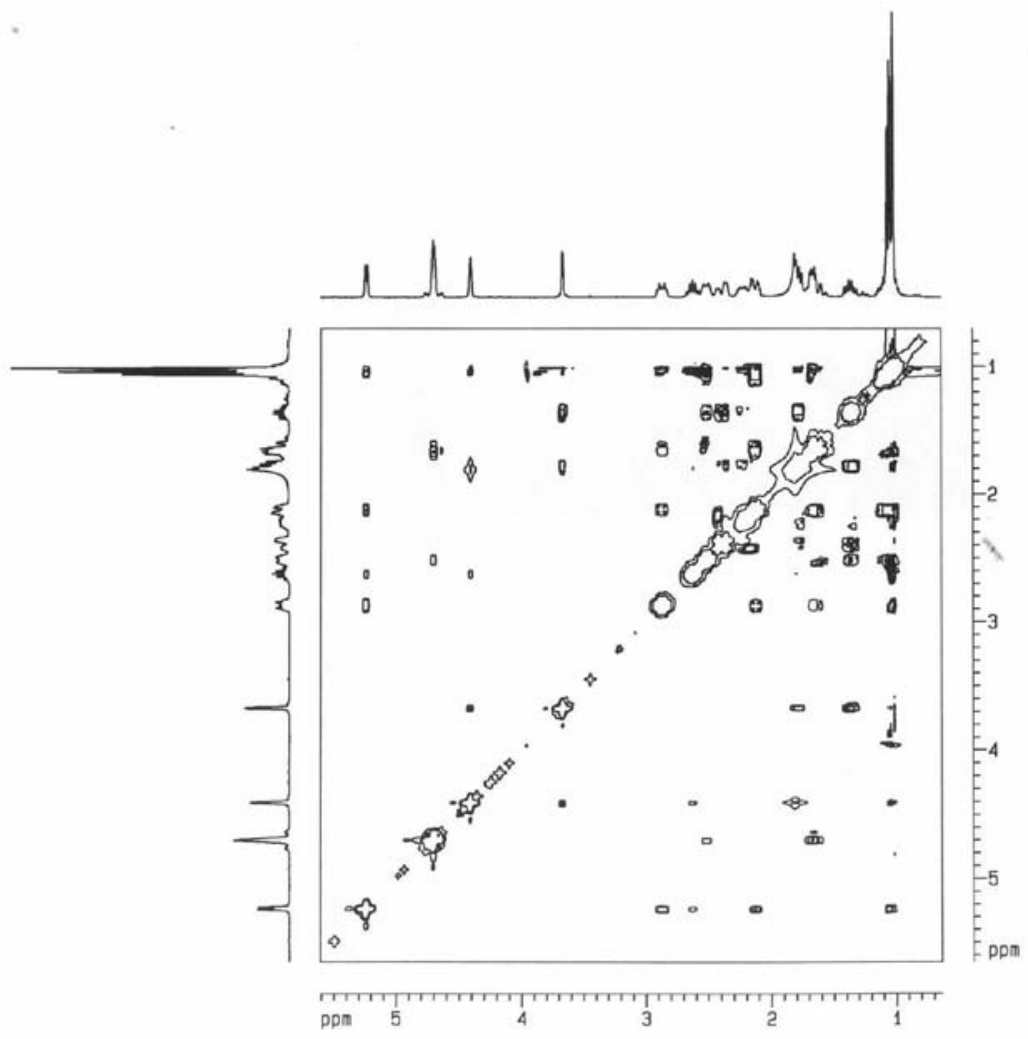

Figure S7. NOESY NMR experiment $\left(\mathrm{CDCl}_{3}, 500 \mathrm{MHz}\right)$ of compound (1) isolated from leaves of Stemodia maritima.

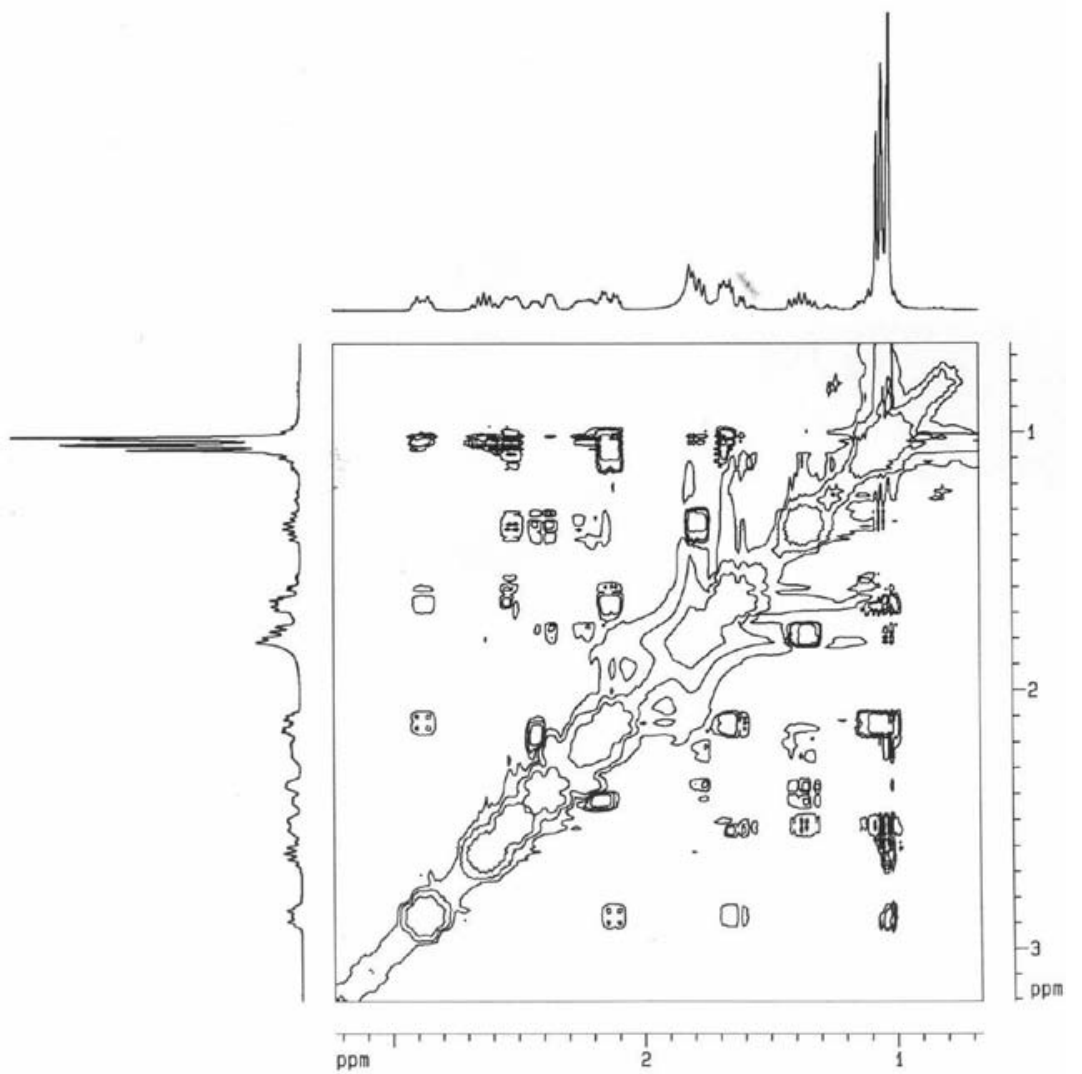

Figure S8. Expansion NOESY NMR experiment $\left(\mathrm{CDCl}_{3}, 500 \mathrm{MHz}\right)$ of compound (1) isolated from leaves of Stemodia maritima. 


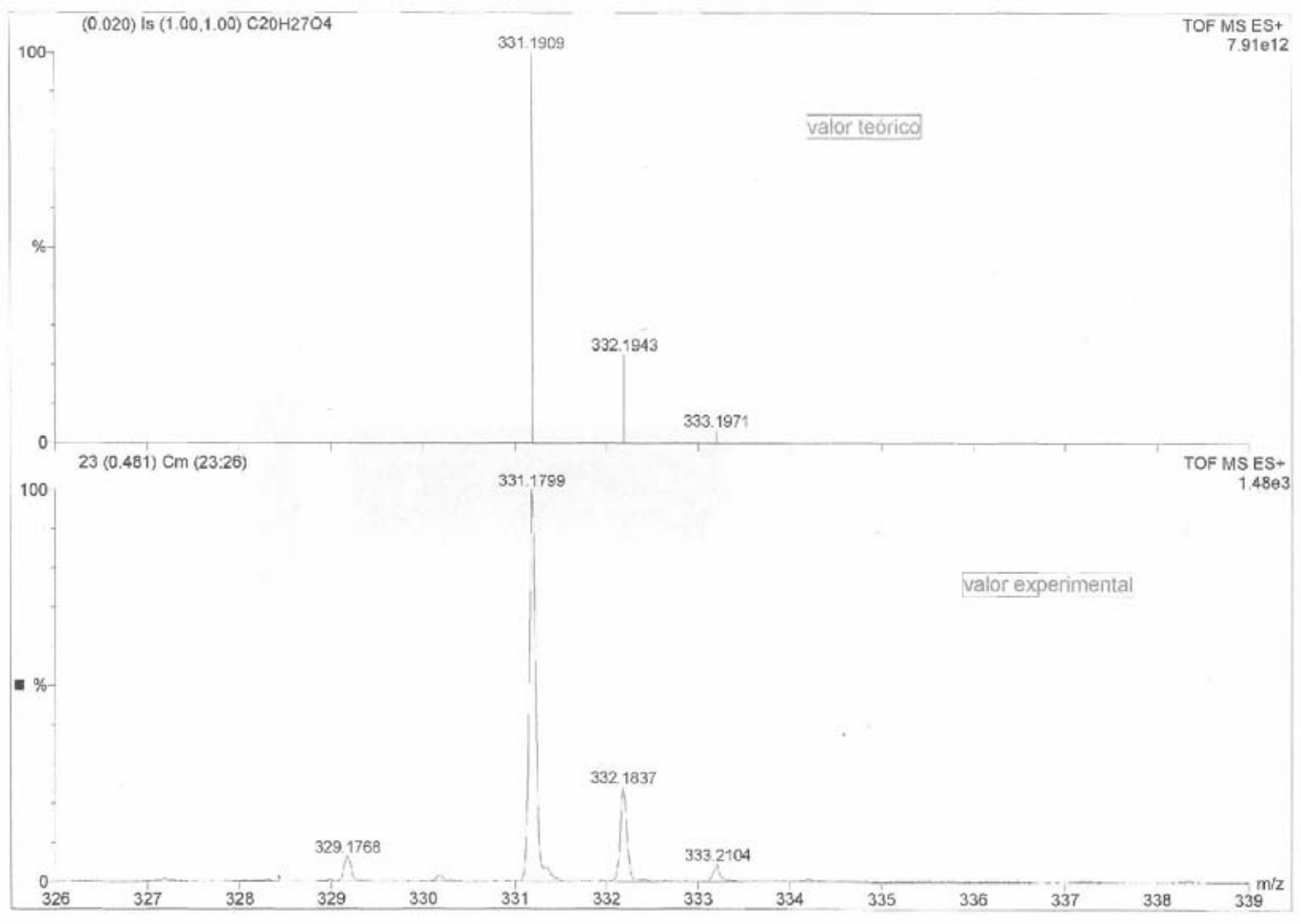

Figure S9. ESI-MS/MS of compound (1) isolated from leaves of Stemodia maritime

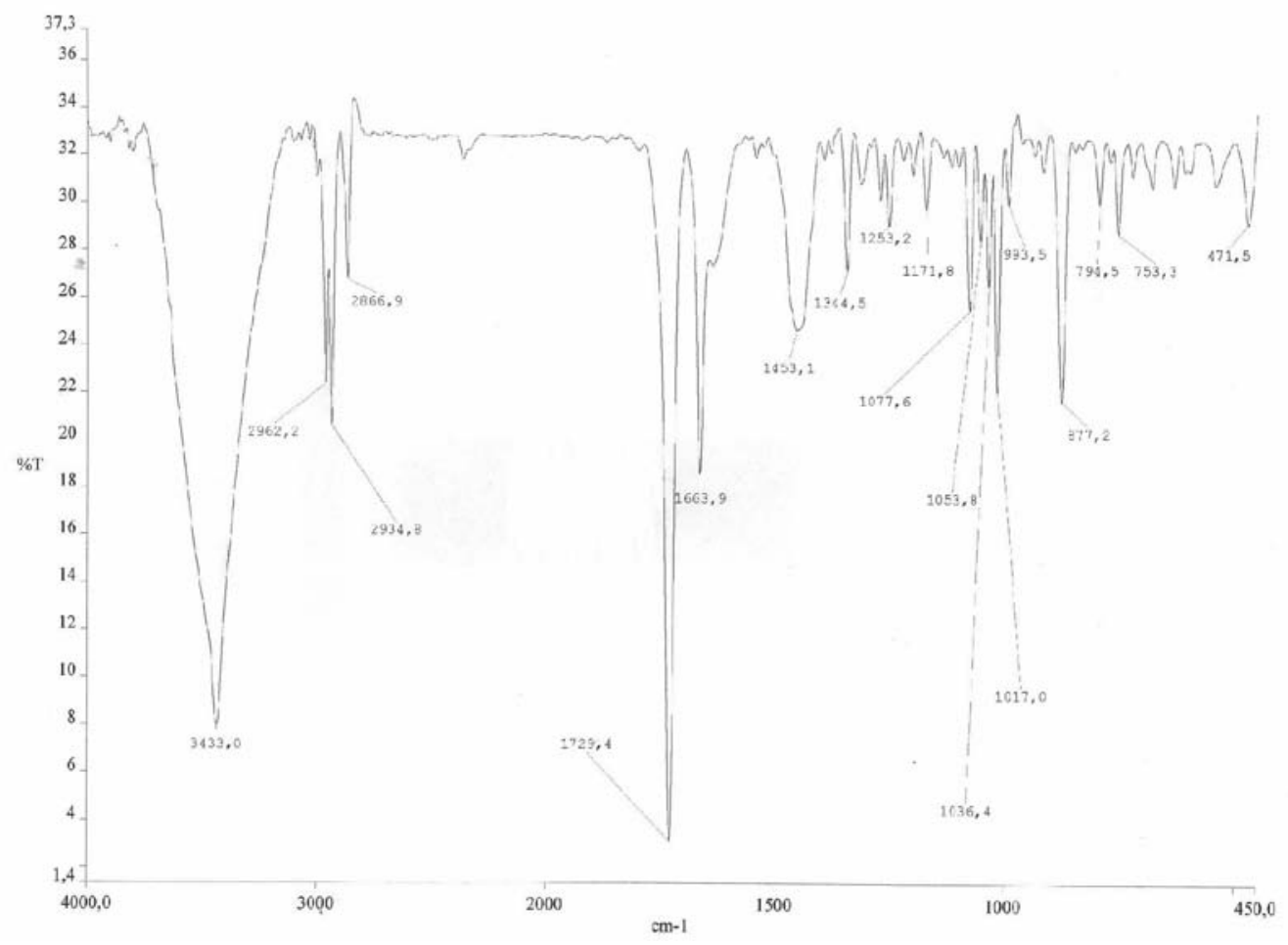

Figure S10. IR spectrum of compound (1) isolated from leaves of Stemodia maritime. 


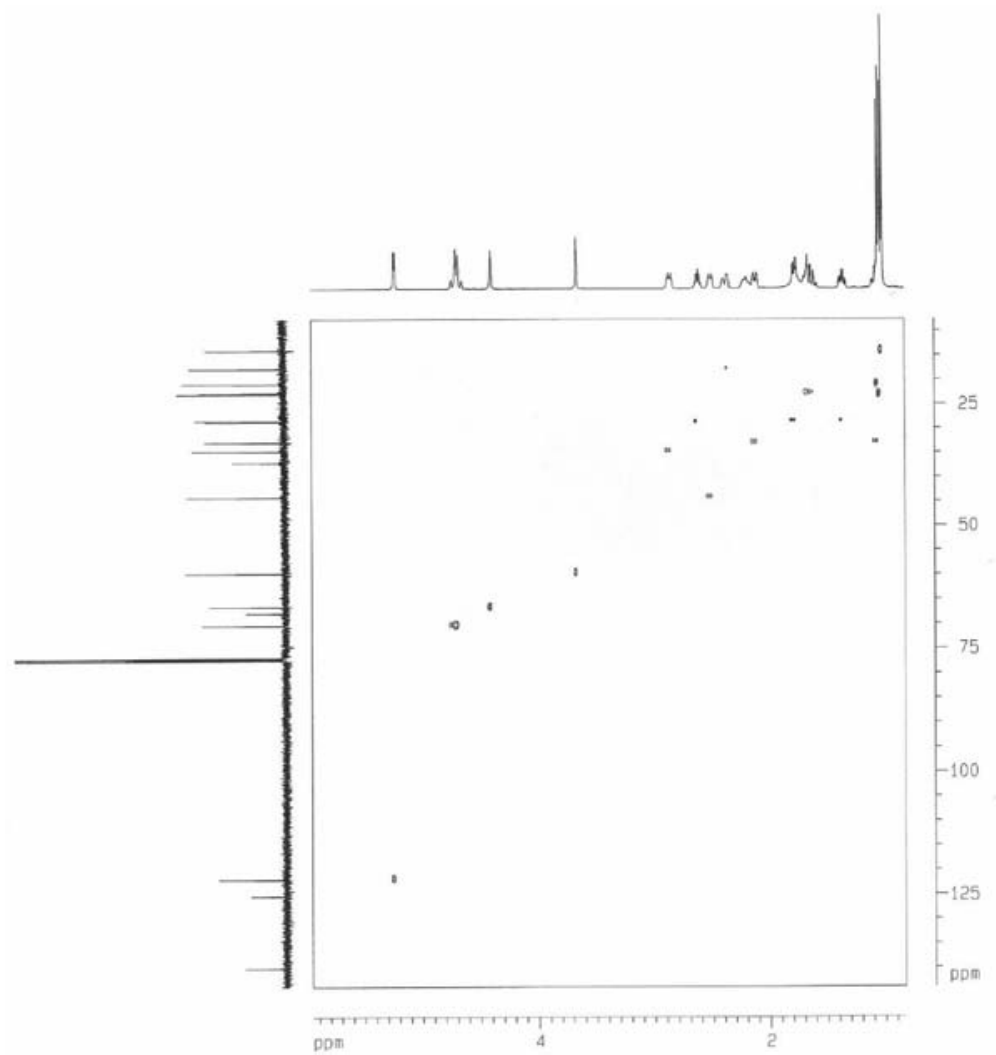

Figure S11. HSQC NMR experiment $\left(\mathrm{CDCl}_{3}, 500 \times 125 \mathrm{MHz}\right)$ of compound (1) isolated from leaves os Stemodia maritima.

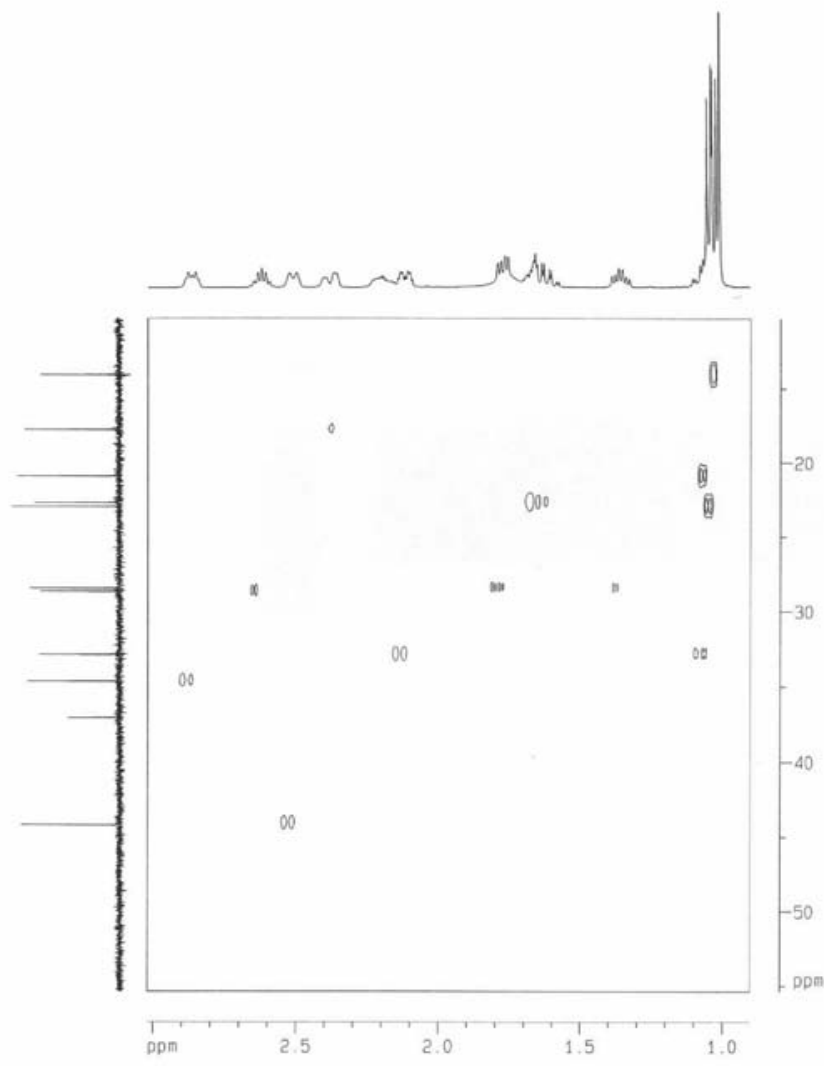

Figure S12. Expansion $1 \mathrm{HSQC}$ NMR experiment $\left(\mathrm{CDCl}_{3}, 500 \times 125 \mathrm{MHz}\right)$ of compound (1) isolated from leaves os Stemodia maritima. 


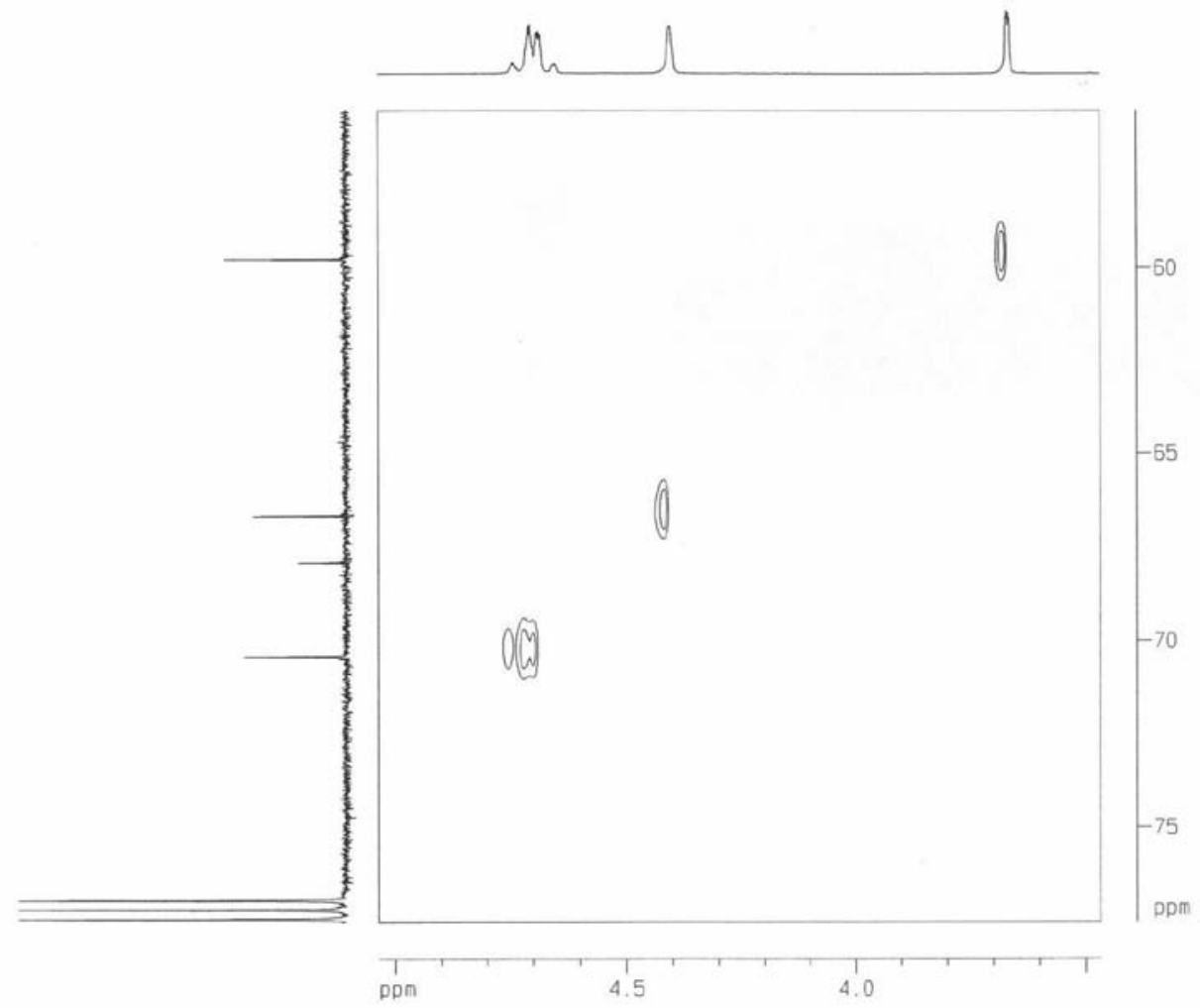

Figure S13. Expansion $2 \mathrm{HSQC}$ NMR experiment $\left(\mathrm{CDCl}_{3}, 500 \times 125 \mathrm{MHz}\right)$ of compound (1) isolated from leaves os Stemodia maritima.

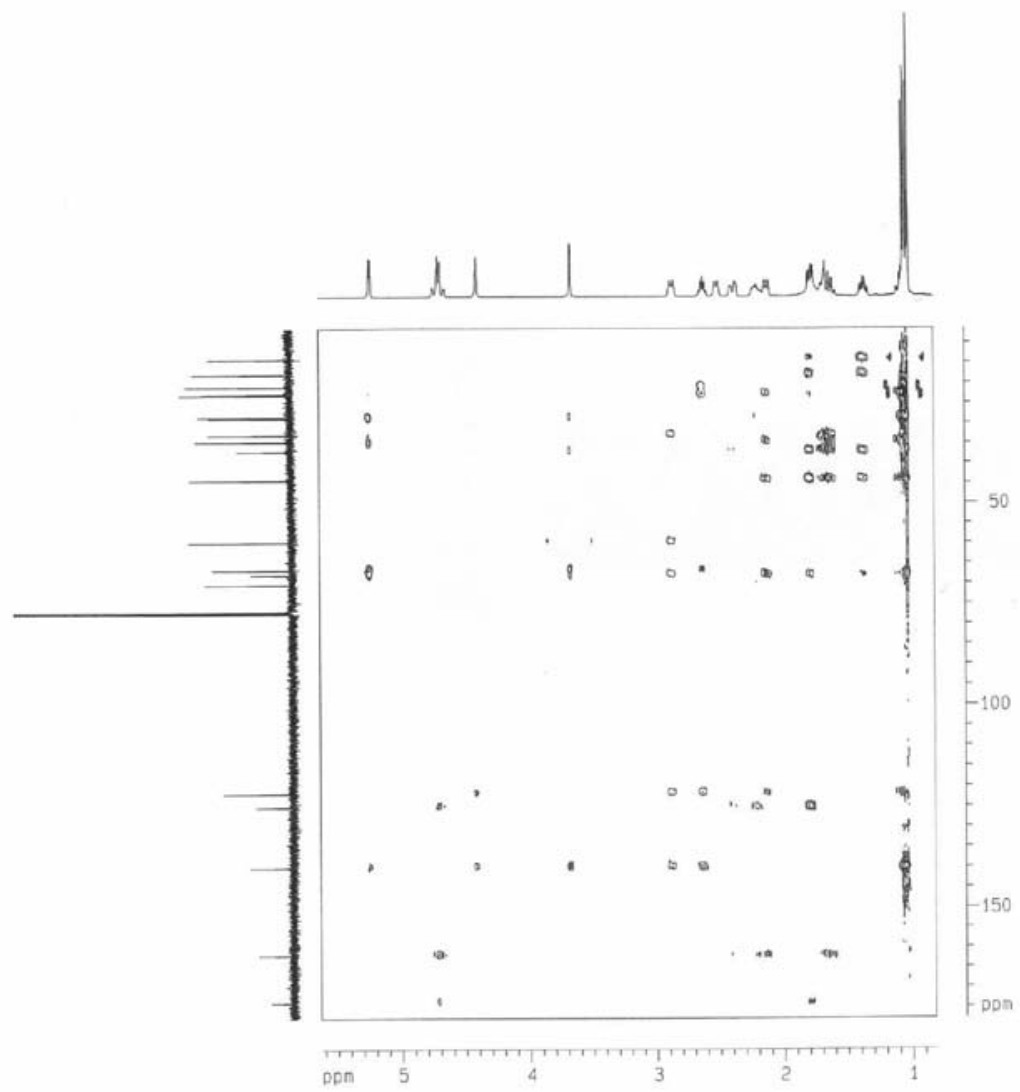

Figure S14. HMBC NMR experiment $\left(\mathrm{CDCl}_{3}, 500 \times 125 \mathrm{MHz}\right)$ of compound (1) isolated from leaves of Stemodia maritima. 


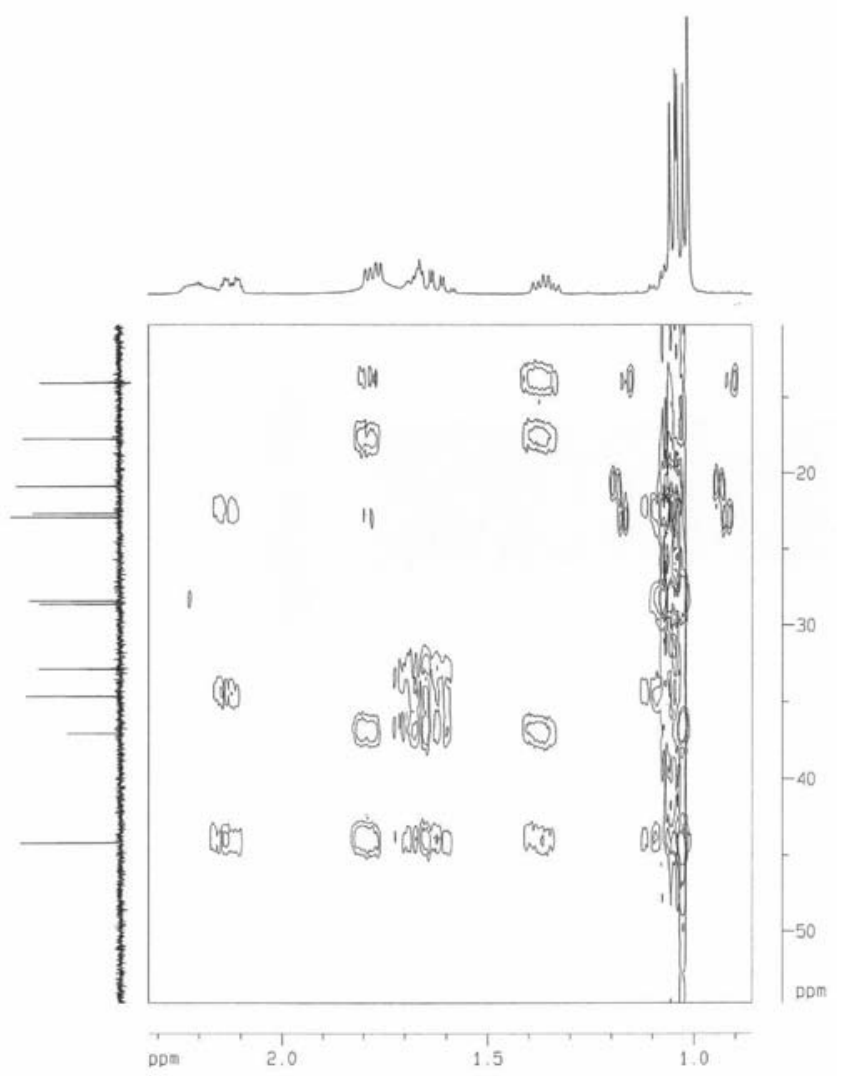

Figure S15. Expansion $1 \mathrm{HMBC}$ NMR experiment $\left(\mathrm{CDCl}_{3}, 500 \times 125 \mathrm{MHz}\right)$ of compound (1) isolated from leaves of Stemodia maritima.

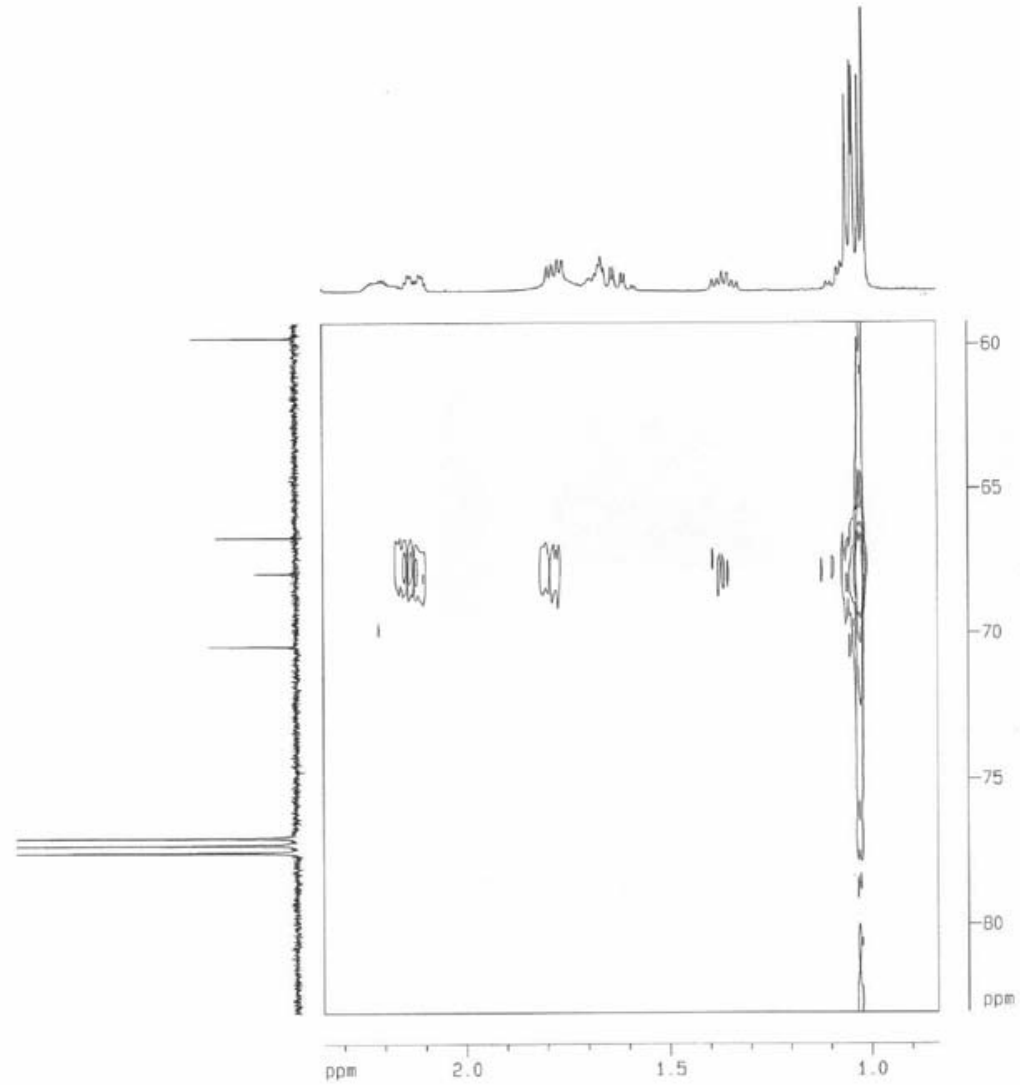

Figure S16. Expansion $2 \mathrm{HMBC}$ NMR experiment $\left(\mathrm{CDCl}_{3}, 500 \times 125 \mathrm{MHz}\right)$ of compound (1) isolated from leaves of Stemodia maritima. 


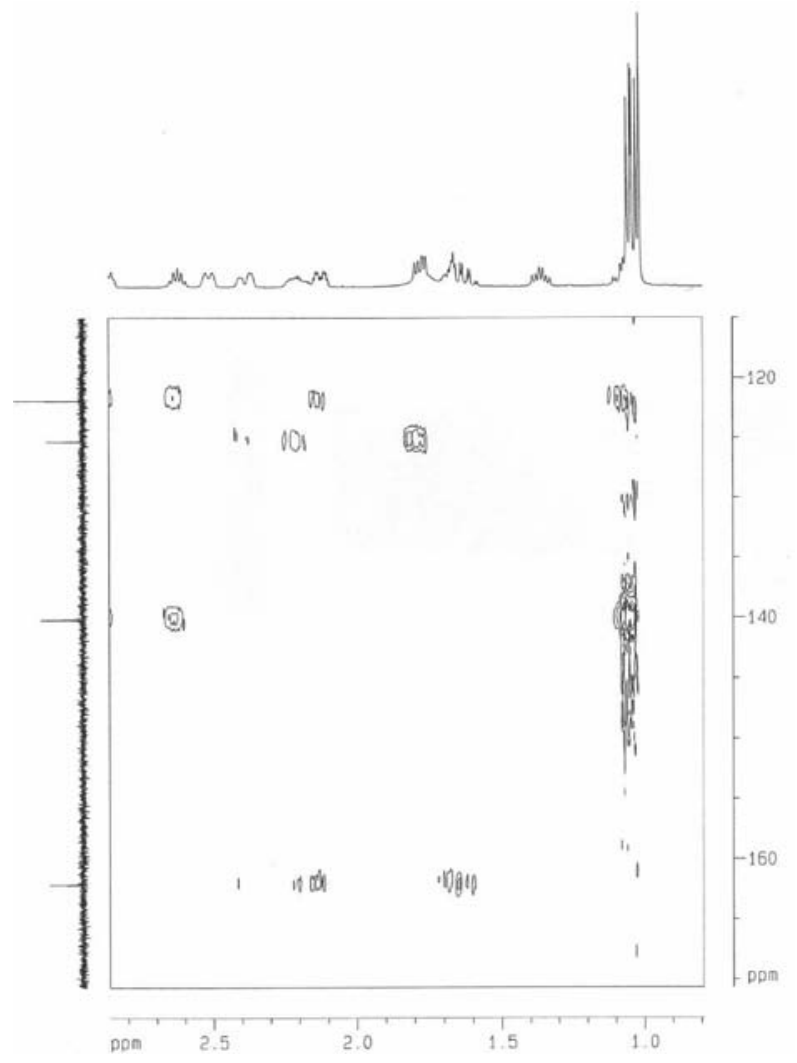

Figure S17. Expansion $3 \mathrm{HMBC}$ NMR experiment $\left(\mathrm{CDCl}_{3}, 500 \times 125 \mathrm{MHz}\right)$ of compound (1) isolated from leaves of Stemodia maritima.

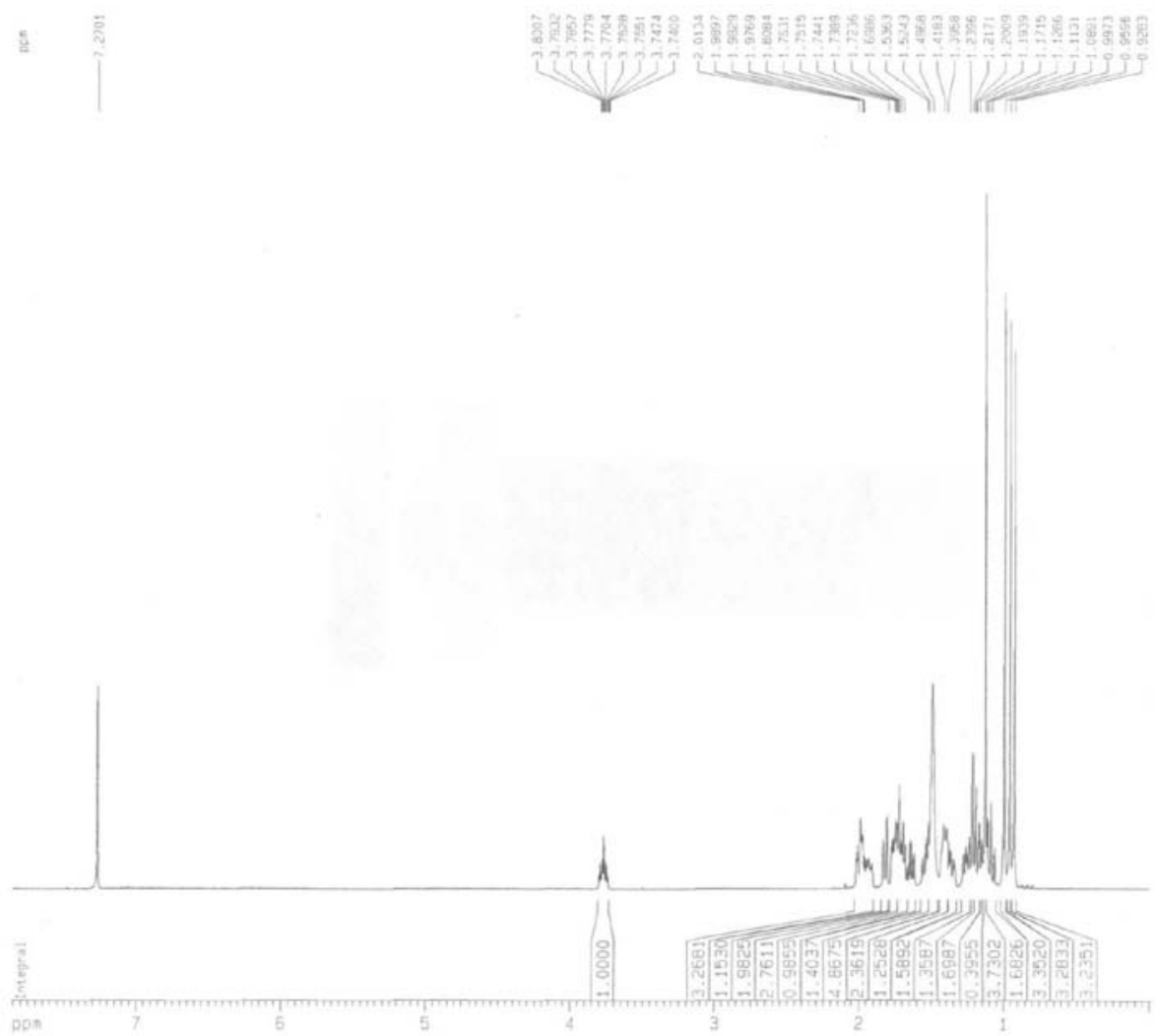

Figure S18. ${ }^{1} \mathrm{H}$ NMR spectrum $\left(\mathrm{CDCl}_{3}, 500 \mathrm{MHz}\right)$ of compound (2) isolated from leaves of Stemodia maritima. 


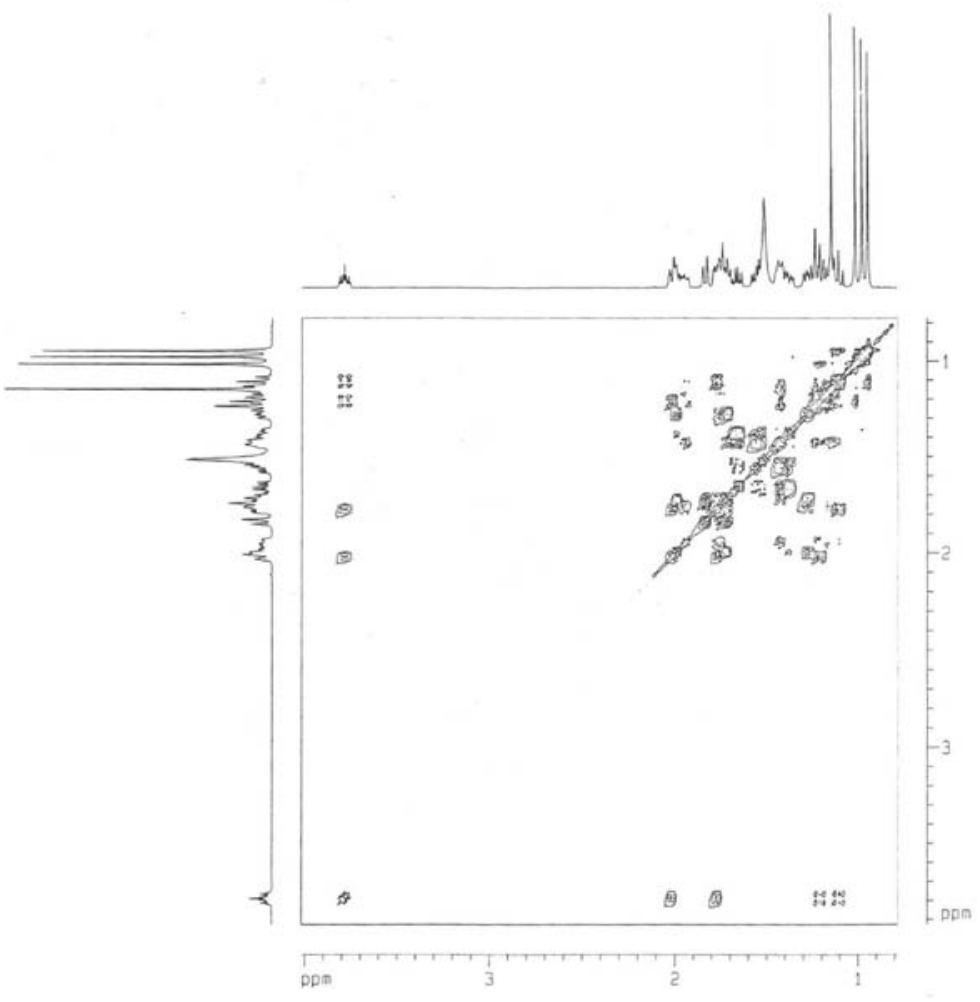

Figure S19. COSY NMR experiment ( $\left.\mathrm{CDCl}_{3}, 500 \mathrm{MHz}\right)$ of compound (2) isolated from leaves of Stemodia maritima.

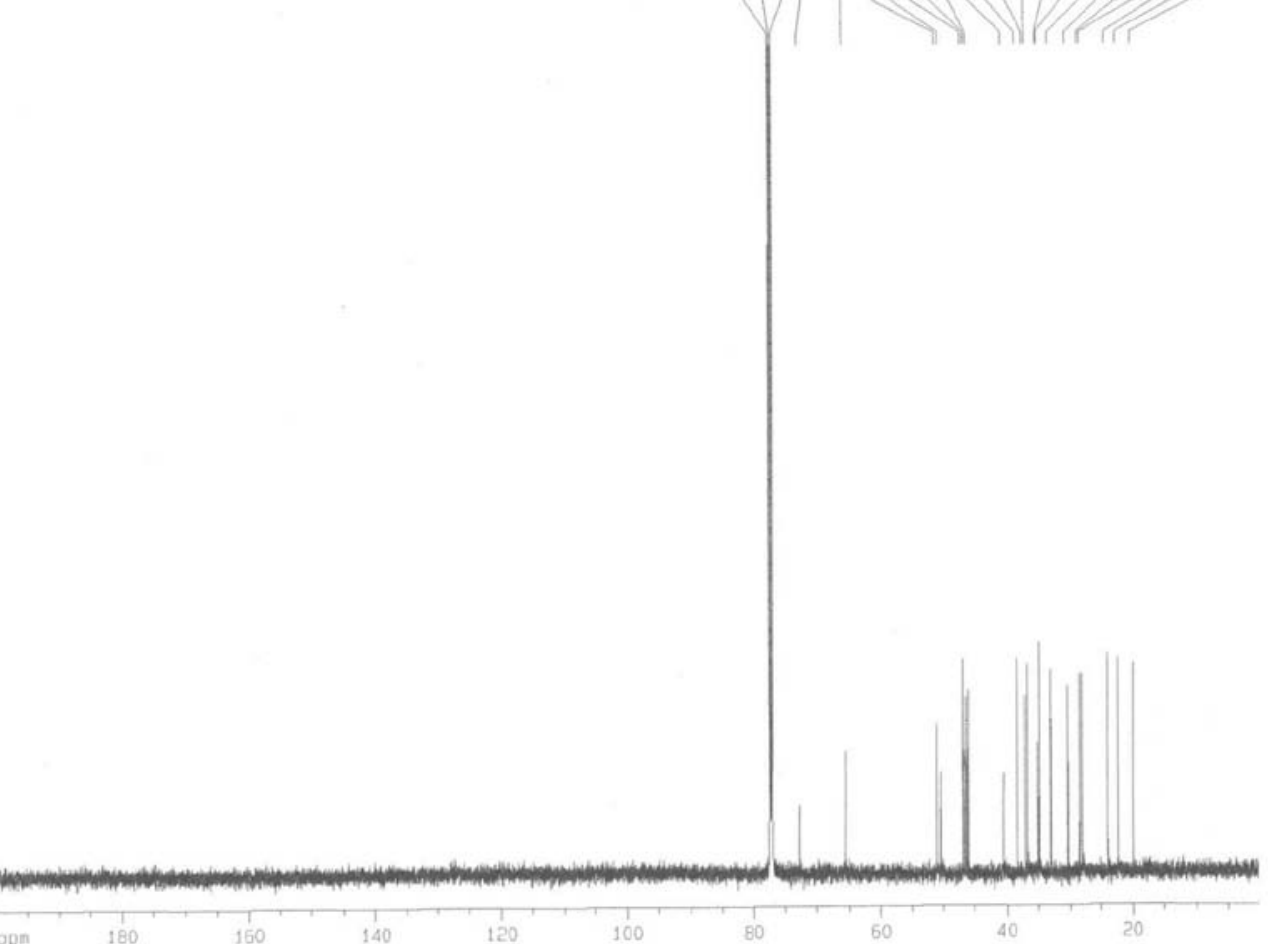

Figure S20. ${ }^{13} \mathrm{C}$ RMN spectrum $\left(\mathrm{CDCl}_{3}, 125 \mathrm{MHz}\right)$ of compound (2) isolated from leaves of Stemodia maritima. 

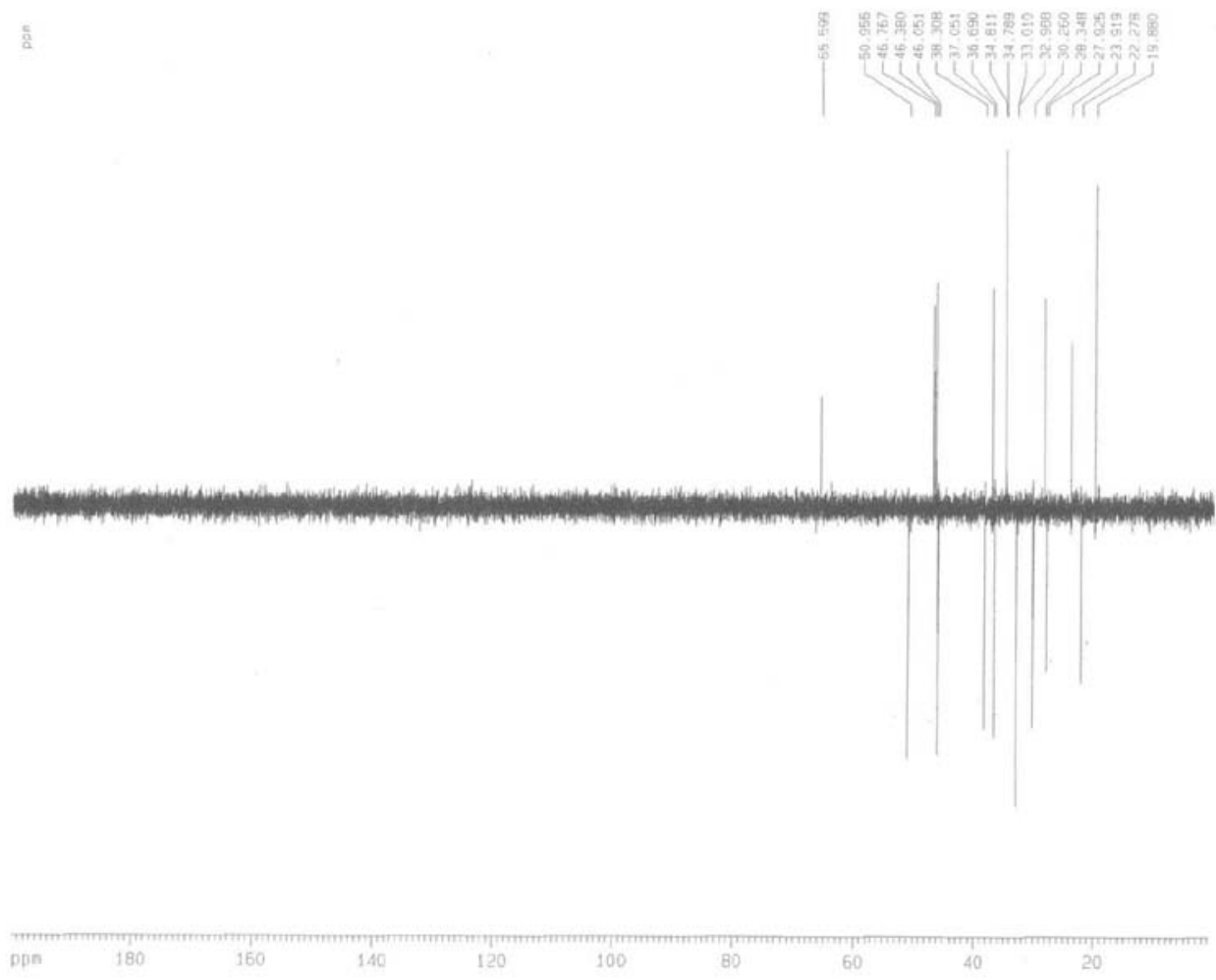

Figure S21. DEPT NMR experiment $\left(\mathrm{CDCl}_{3}, 125 \mathrm{MHz}\right)$ of compound (2) isolated from leaves of Stemodia maritima.

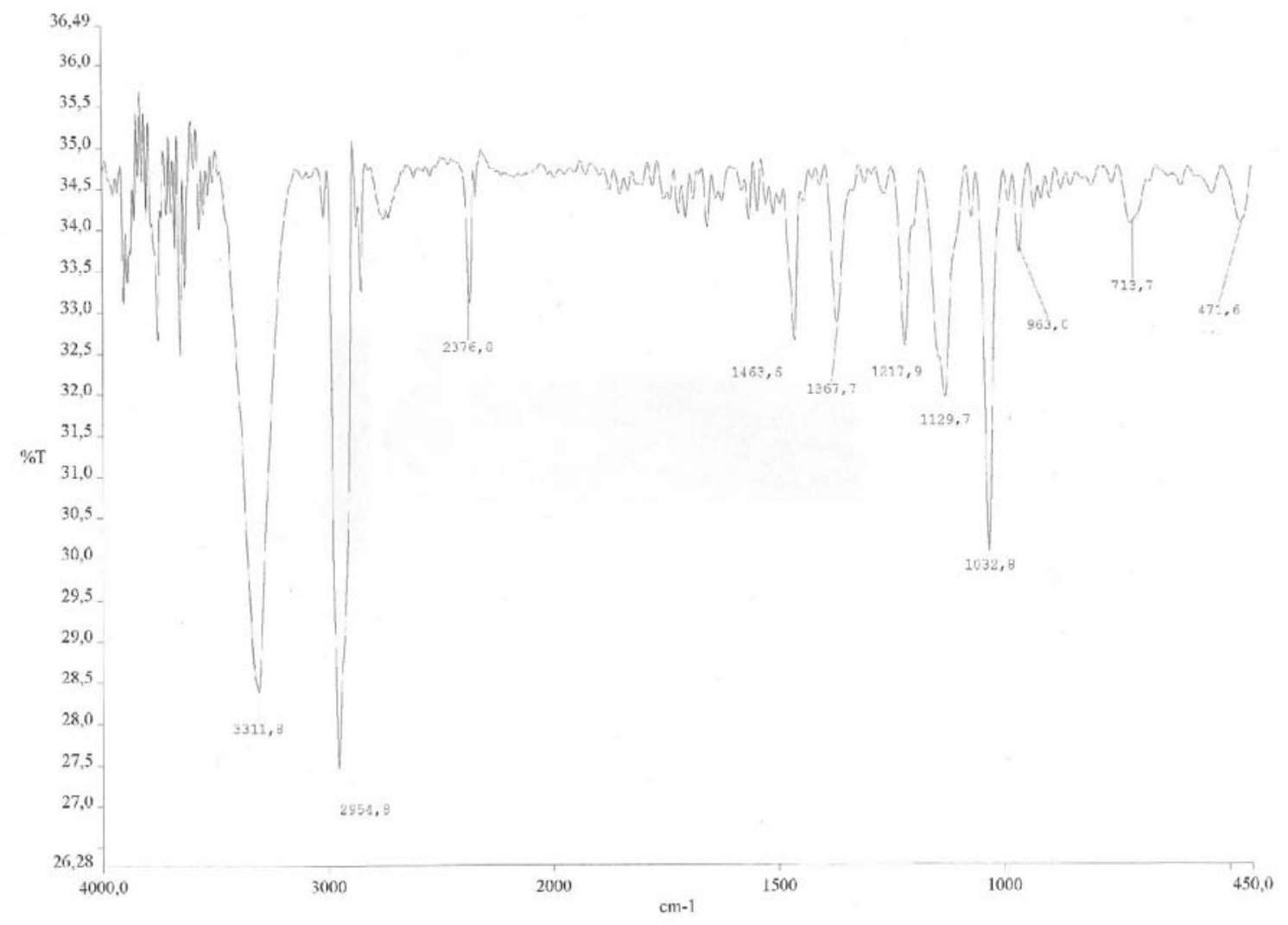

Figure S22. IR spectrum of compound (2) isolated from leaves of Stemodia marítima 


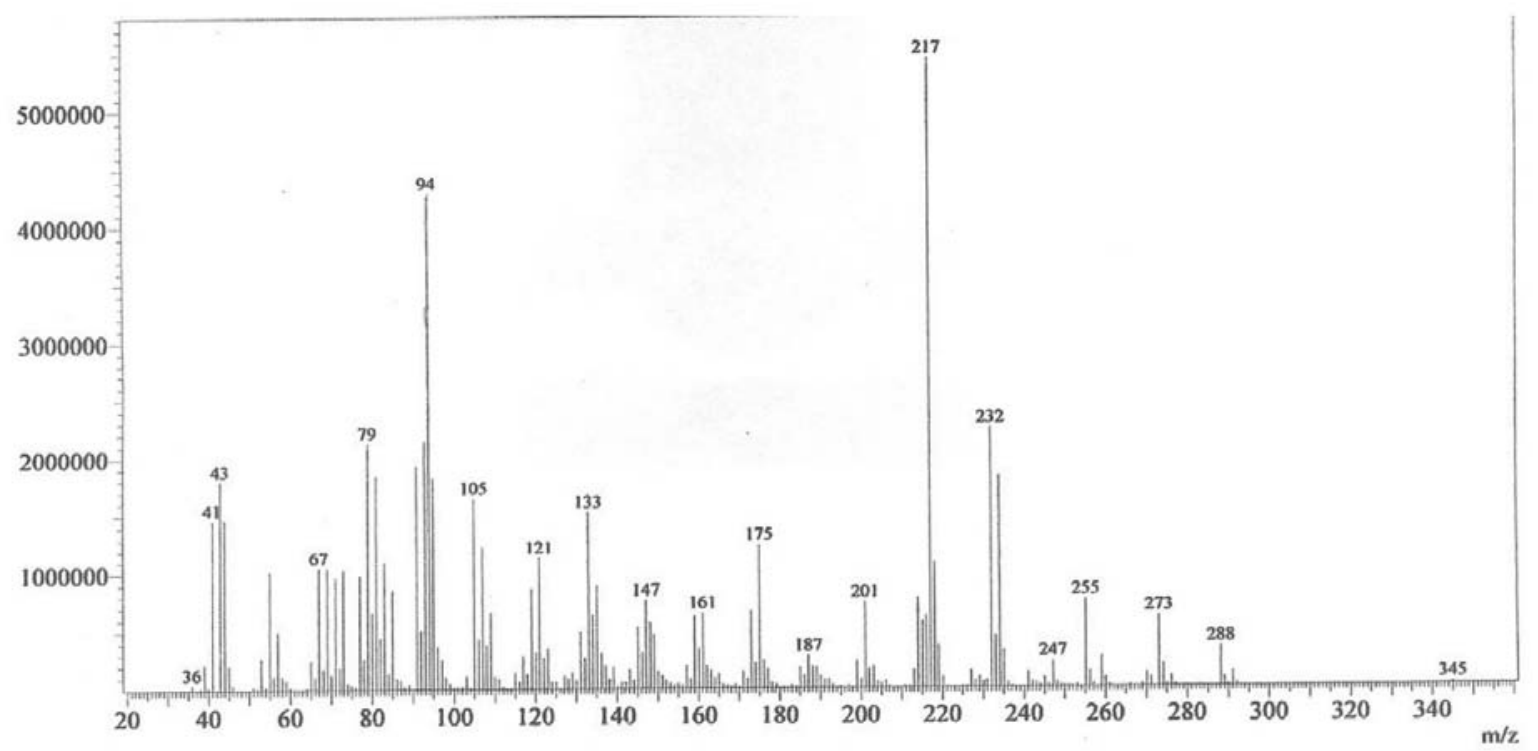

Figure S23. MS of compound (2) isolated from leaves of Stemodia maritima.

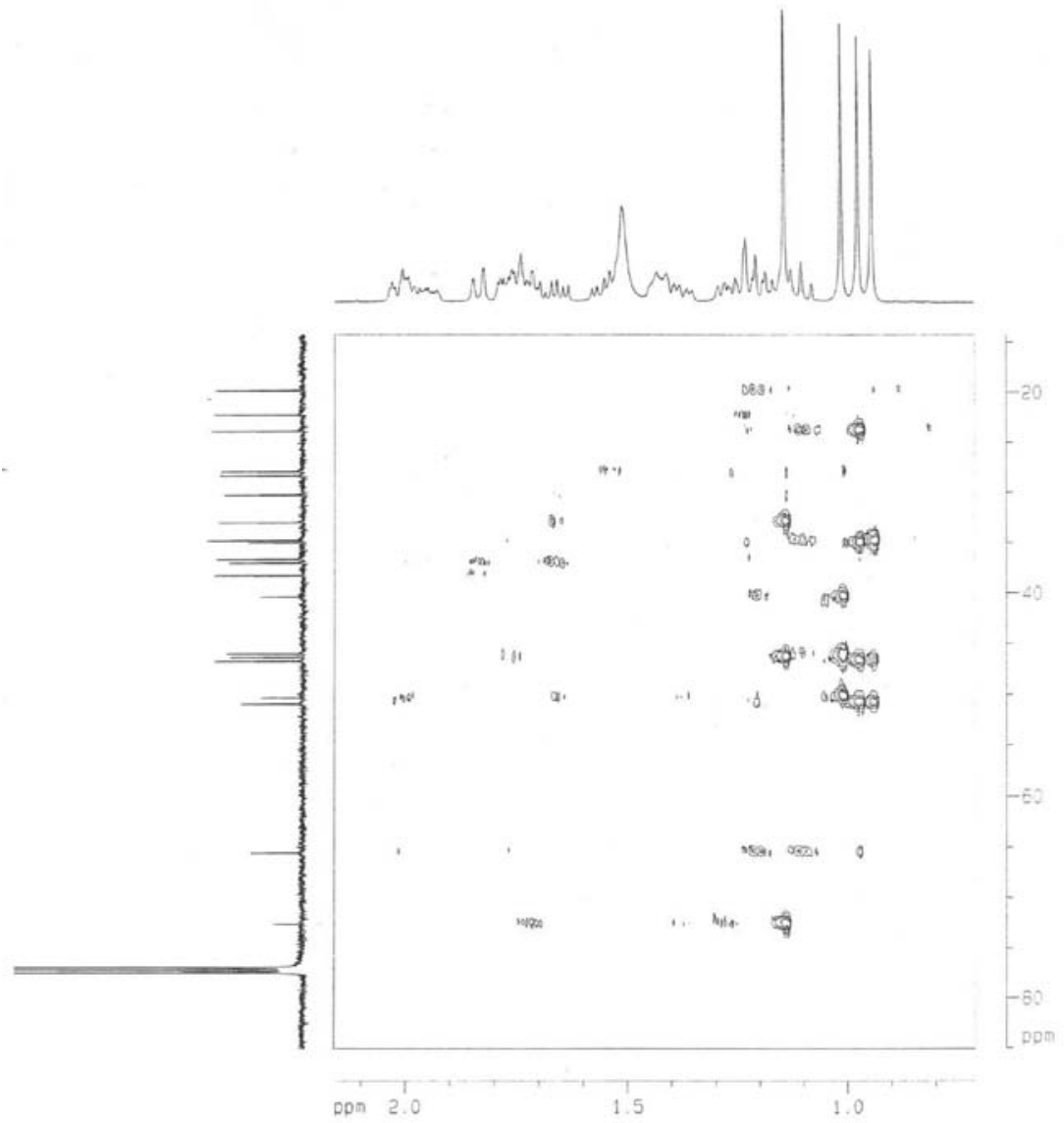

Figure S24. HMBC NMR experiment $\left(\mathrm{CDCl}_{3}, 500 \times 125 \mathrm{MHz}\right)$ of compound (2) isolated from leaves of Stemodia maritima. 


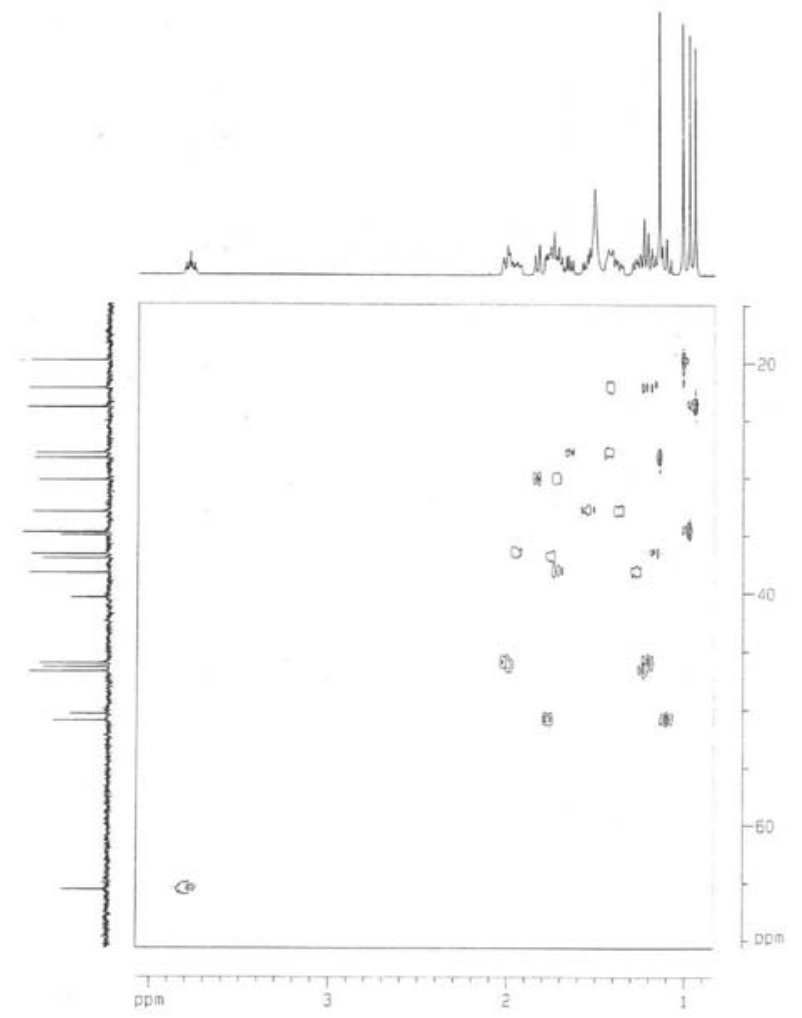

Figure S25. HSQC NMR experiment $\left(\mathrm{CDCl}_{3}, 500 \times 125 \mathrm{MHz}\right)$ of compound (2) isolated from leaves of Stemodia maritima.

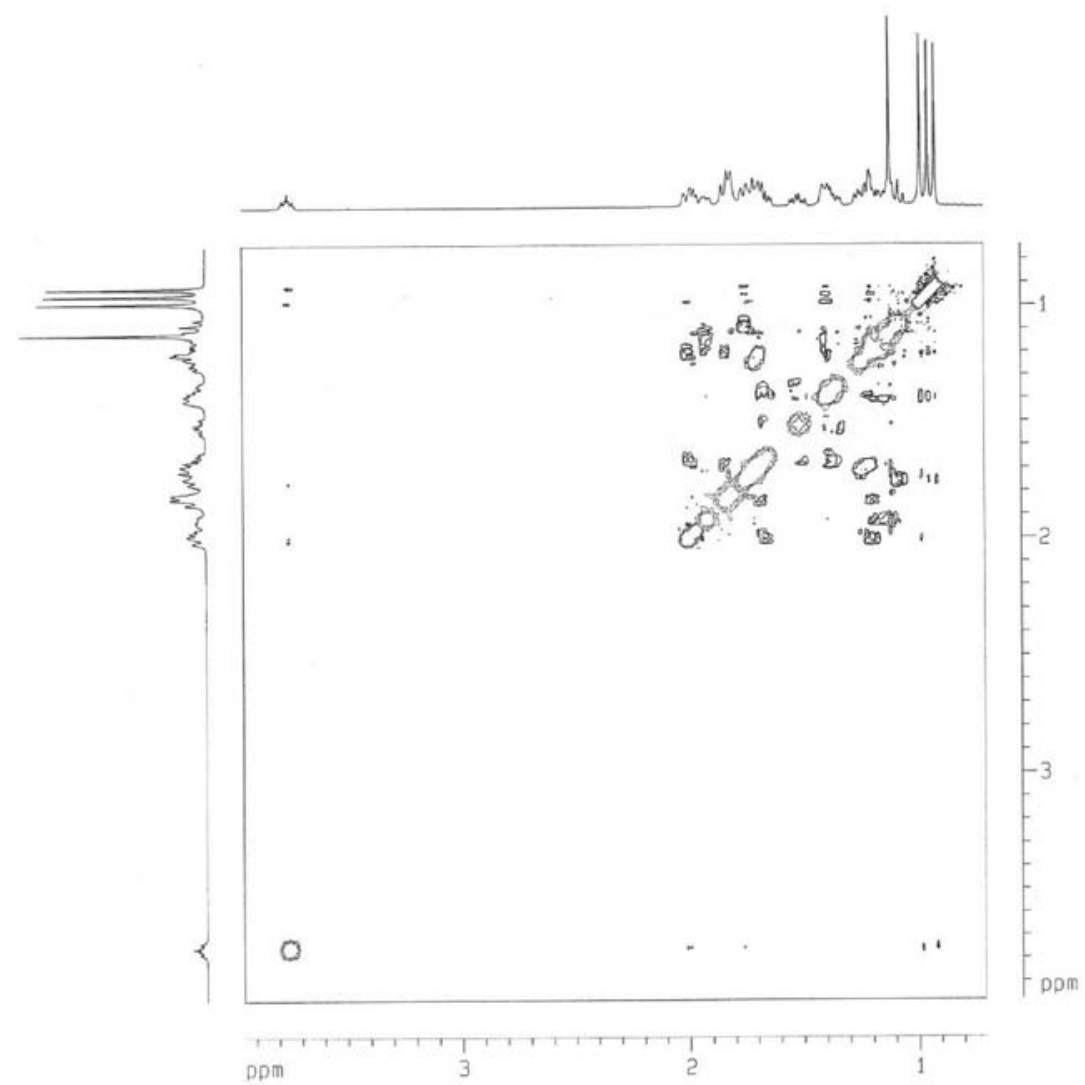

Figure S26. NOESY NMR experiment $\left(\mathrm{CDCl}_{3}, 500 \mathrm{MHz}\right)$ of compound (2) isolated from leaves of Stemodia maritima. 


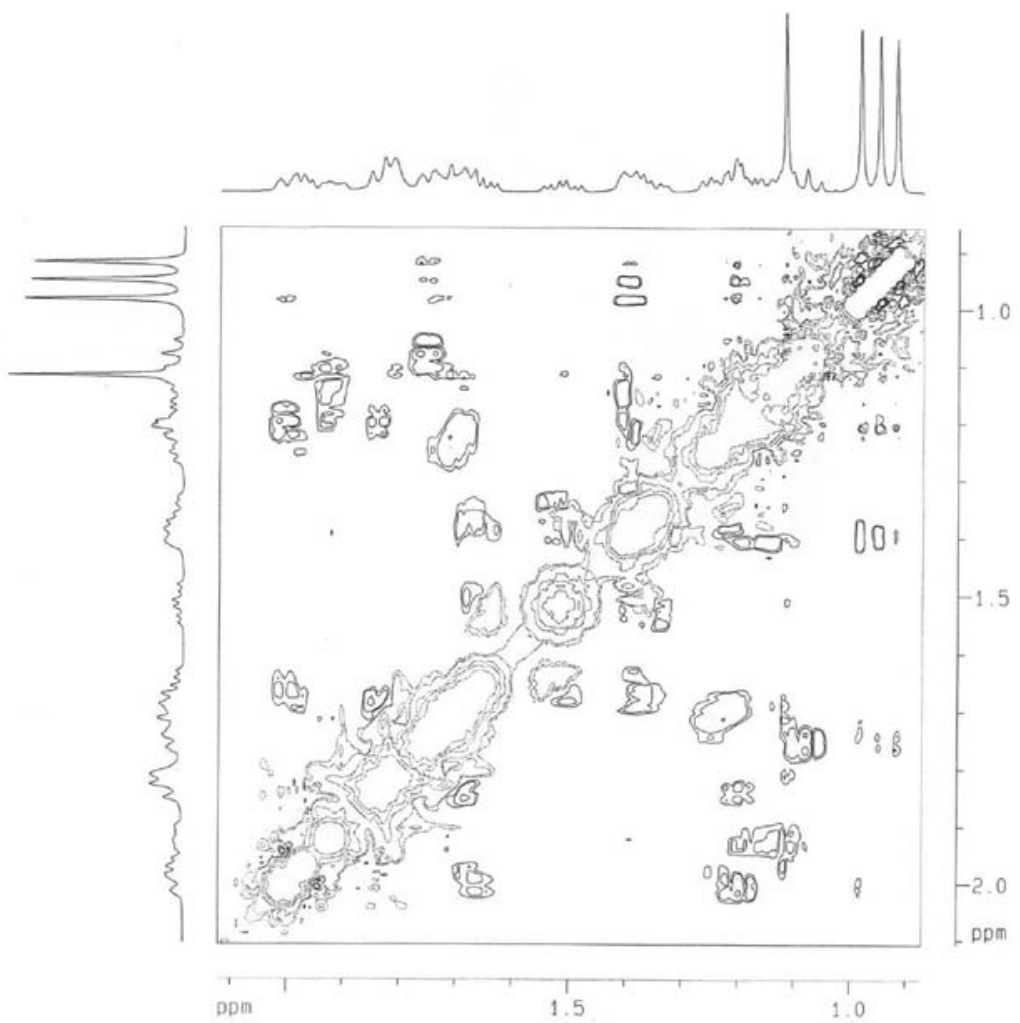

Figure S27. Expansion NOESY NMR experiment $\left(\mathrm{CDCl}_{3}, 500 \mathrm{MHz}\right)$ of compound (2) isolated from leaves of Stemodia maritima.

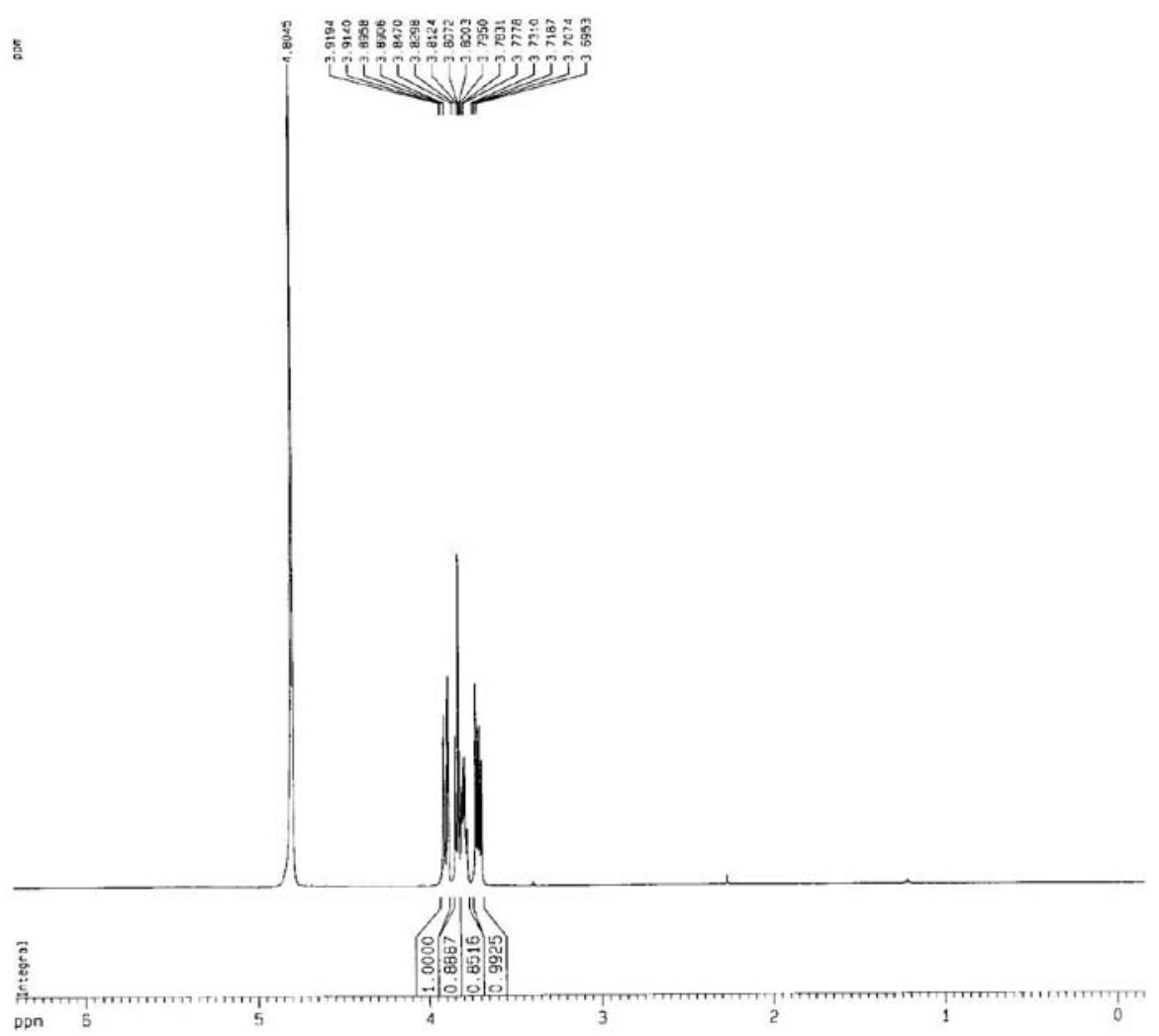

Figure S28. ${ }^{~} \mathrm{H}$ NMR spectrum $\left(\mathrm{CDCl}_{3}, 500 \mathrm{MHz}\right)$ of D-mannitol isolated from stems of Stemodia maritima. 


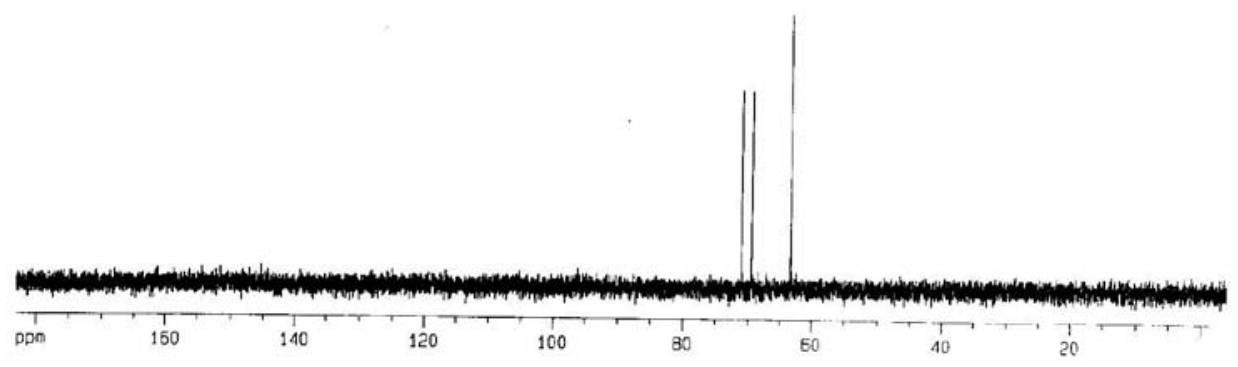

Figure S29 ${ }^{13} \mathrm{C}$ NMR spectrum $\left(\mathrm{CDCl}_{3}, 125 \mathrm{MHz}\right)$ of D-mannitol isolated from stems of Stemodia maritima.

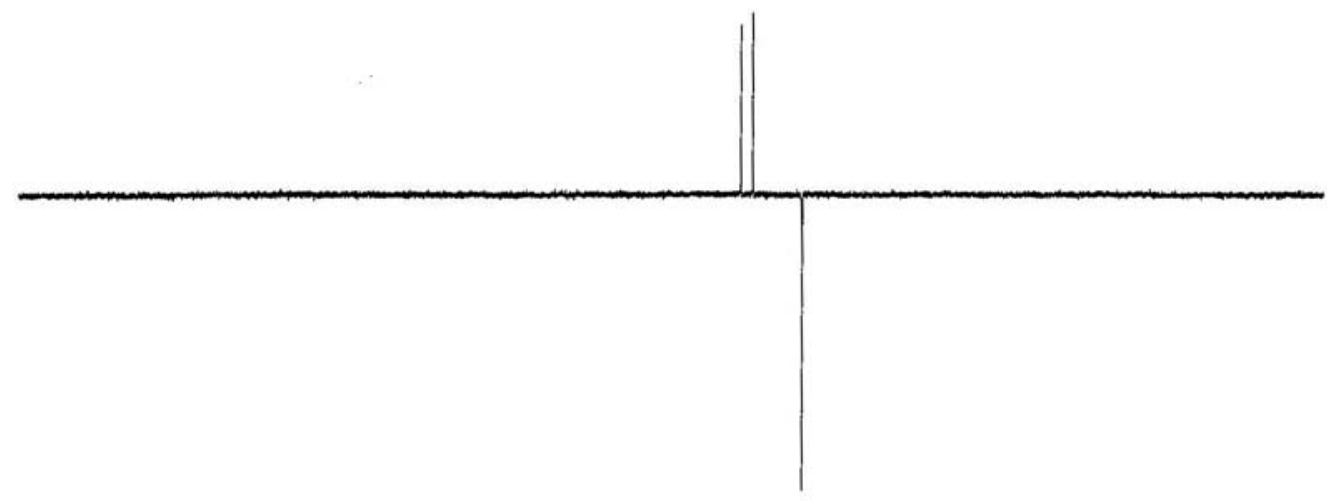

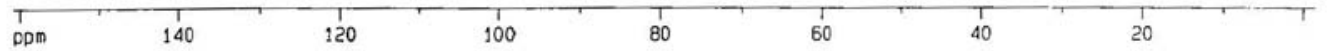

Figure S30. DEPT NMR experiment $\left(\mathrm{CDCl}_{3}, 125 \mathrm{MHz}\right)$ of D-mannitol isolated from stems of Stemodia maritima. 
ฐ
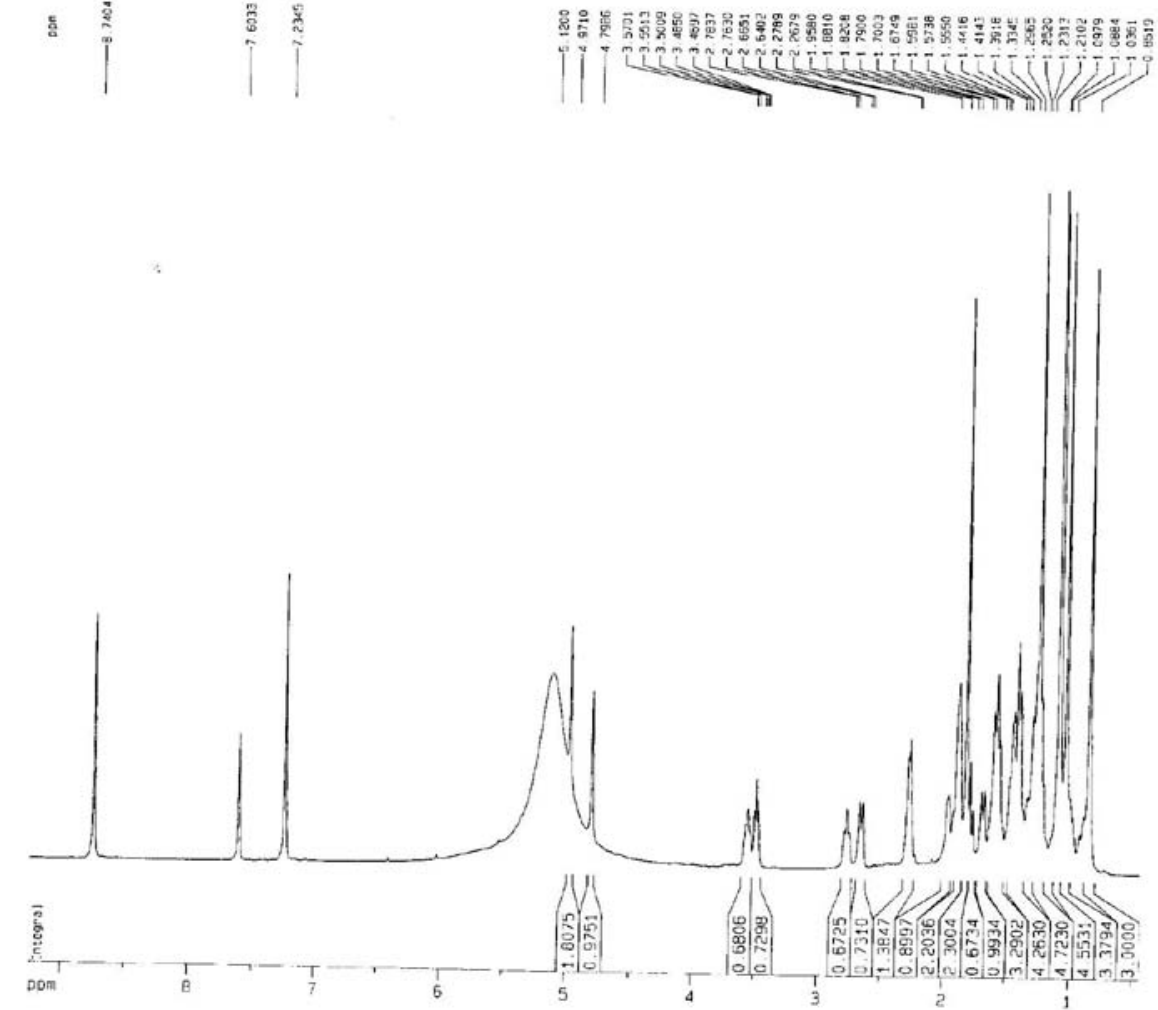

Figure S31. ${ }^{1} \mathrm{H}$ NMR spectrum $\left(\mathrm{C}_{5} \mathrm{D}_{5} \mathrm{~N}, 500 \mathrm{MHz}\right)$ of betulinic acid isolated from stems of Stemodia maritima.

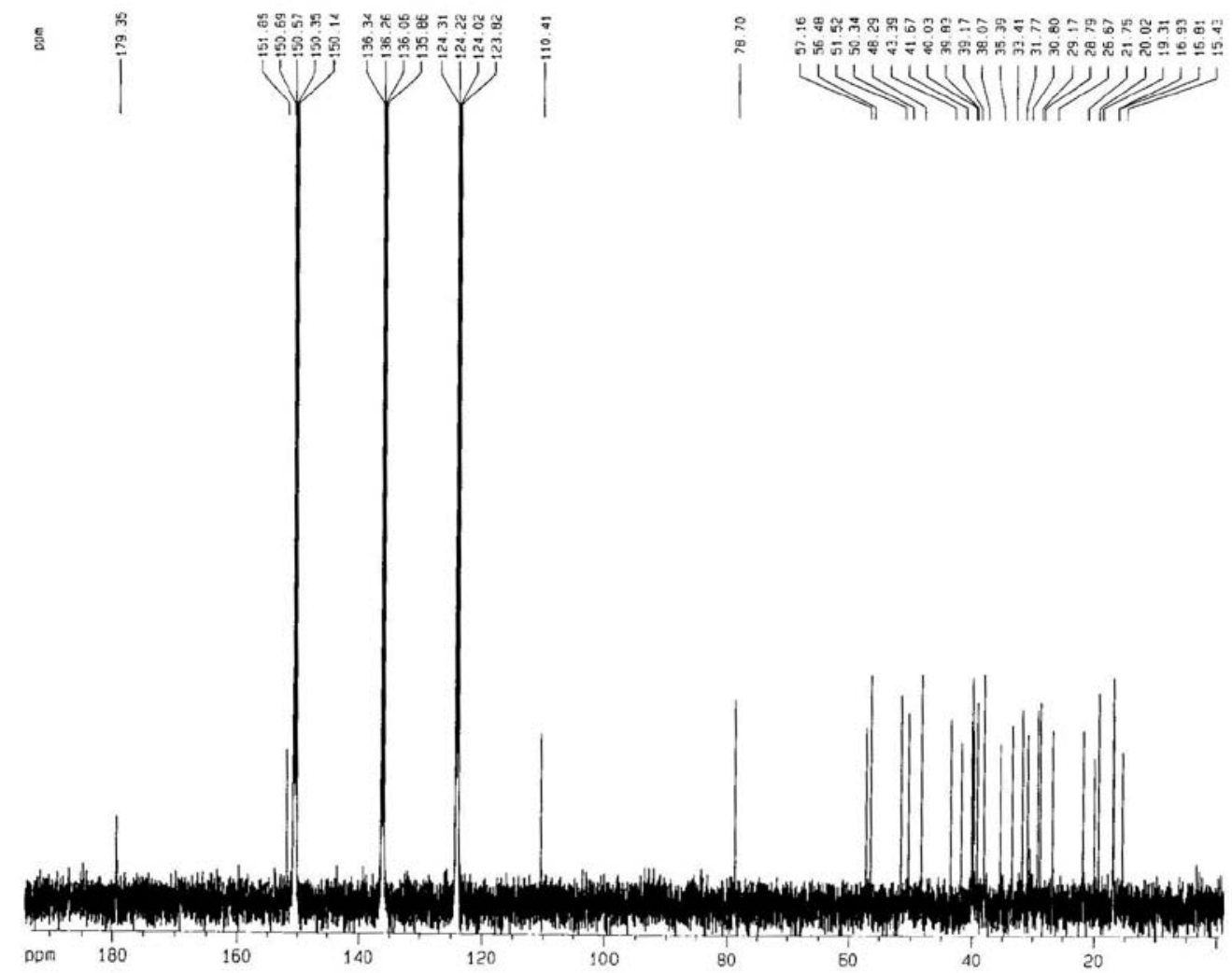

Figure S32. ${ }^{13} \mathrm{C}$ RMN spectrum $\left(\mathrm{C}_{5} \mathrm{D}_{5} \mathrm{~N}, 125 \mathrm{MHz}\right)$ of betulinic acid isolated from stems of Stemodia maritima. 
๕.

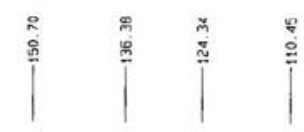

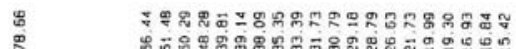
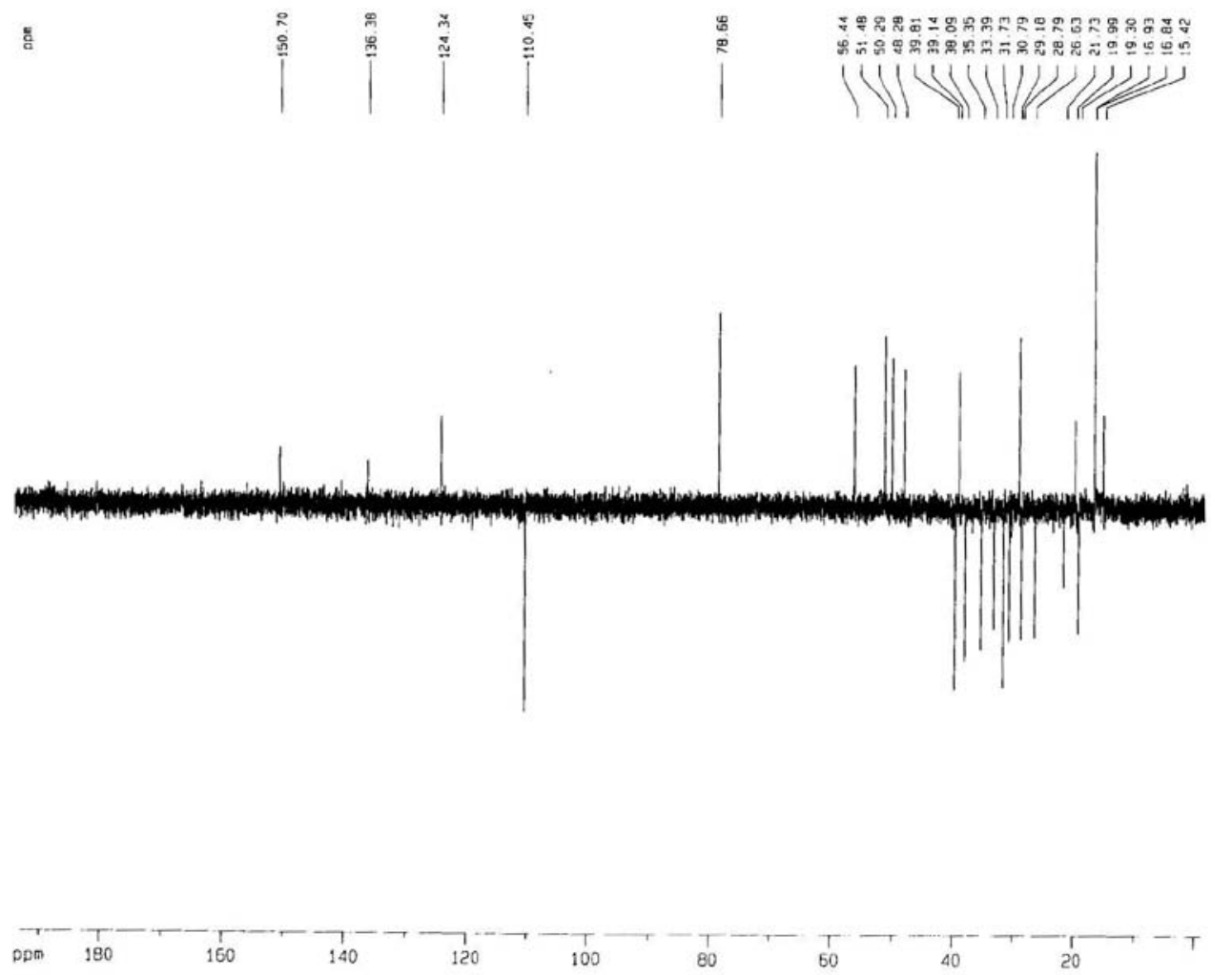

Figure S33. DEPT NMR experiment $\left(\mathrm{C}_{5} \mathrm{D}_{5} \mathrm{~N}, 125 \mathrm{MHz}\right)$ of betulinic acid isolated from stems of Stemodia maritima.

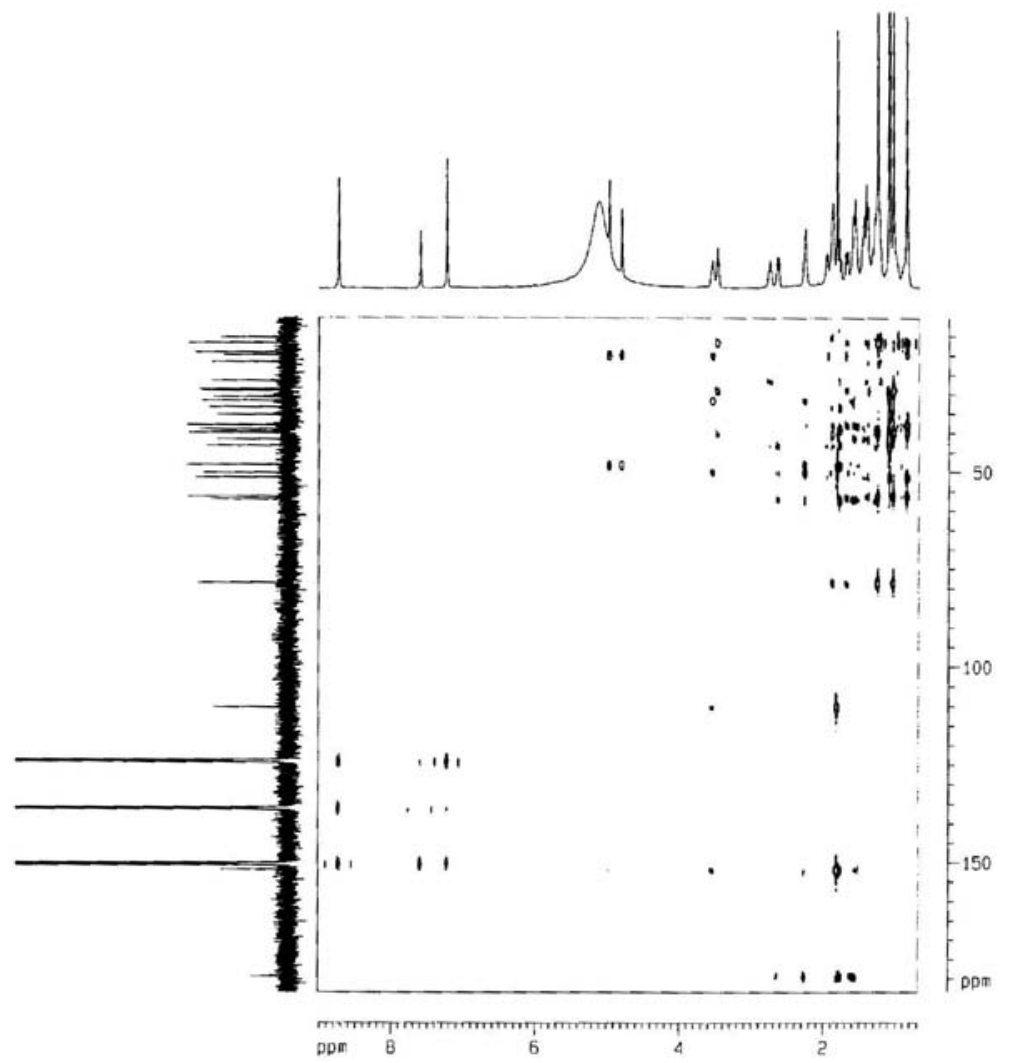

Figure S34. HMBC NMR experiment $\left(\mathrm{C}_{5} \mathrm{D}_{5} \mathrm{~N}, 500 \times 125 \mathrm{MHz}\right)$ of betulinic acid isolated from stems of Stemodia maritima. 


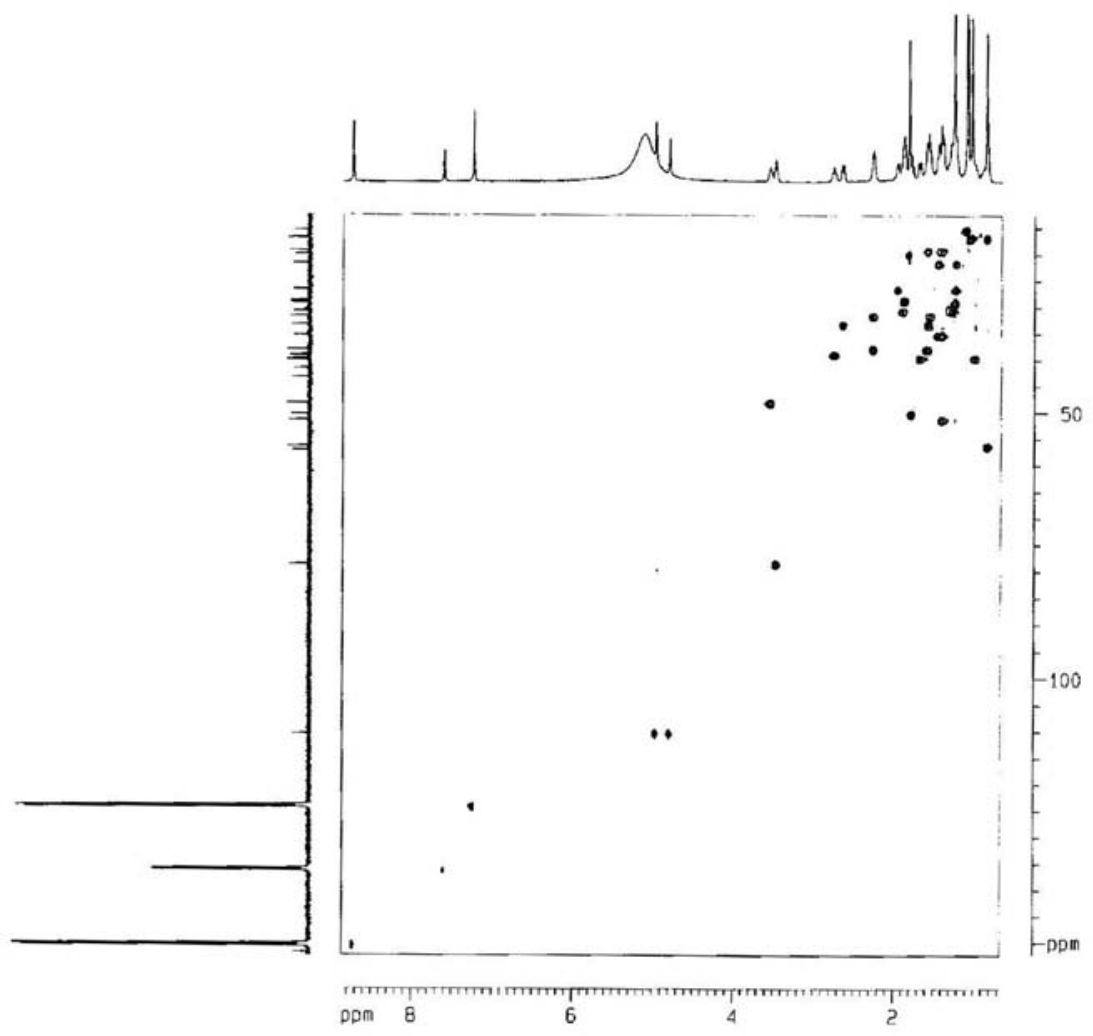

Figure S35. HSQC NMR experiment $\left(\mathrm{C}_{5} \mathrm{D}_{5} \mathrm{~N}, 500 \times 125 \mathrm{MHz}\right)$ of betulinic acid isolated from stems of Stemodia maritima.
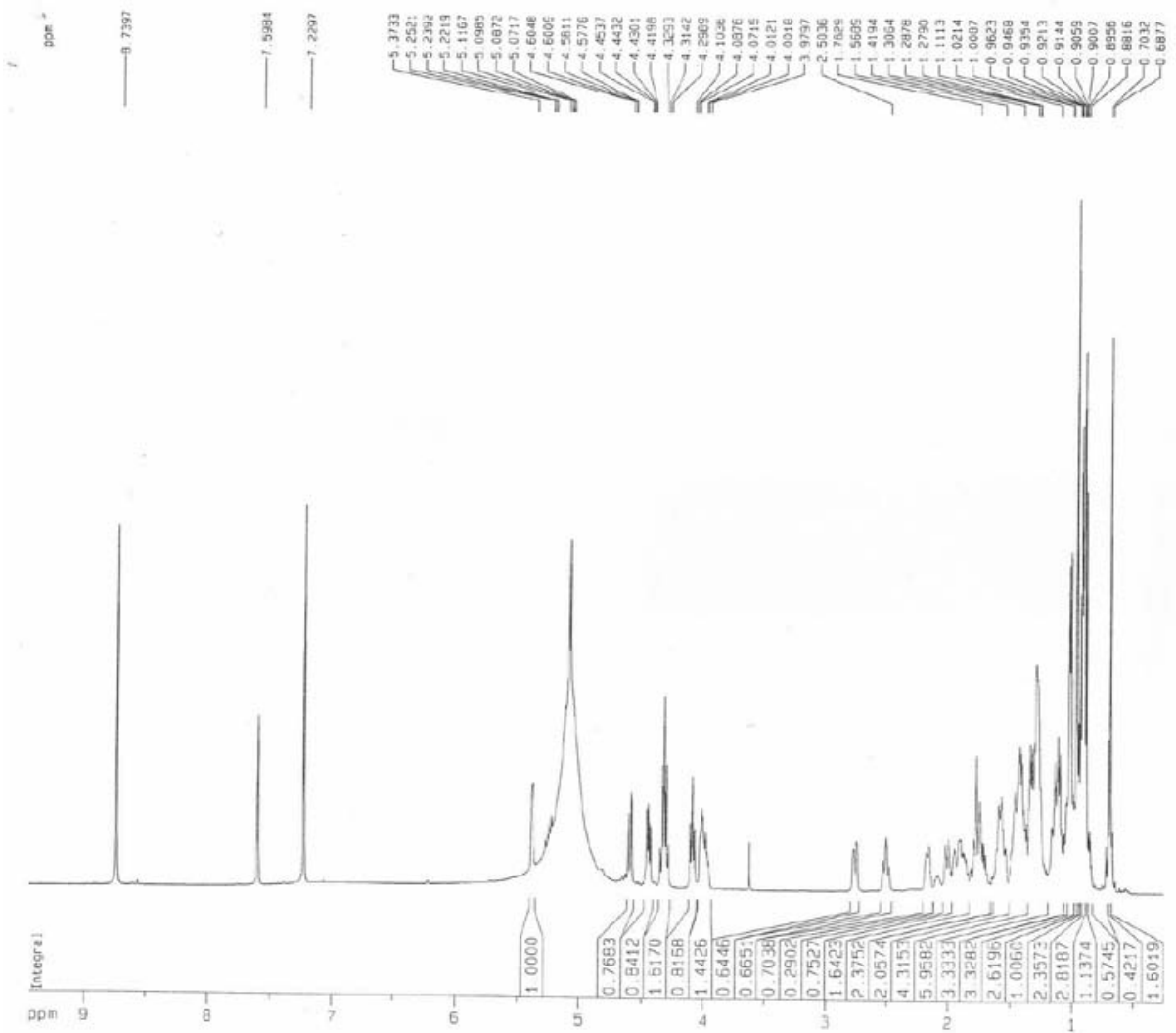

Figure S36. ${ }^{1} \mathrm{H}$ NMR spectrum $\left(\mathrm{C}_{5} \mathrm{D}_{5} \mathrm{~N}, 500 \mathrm{MHz}\right)$ of steroidal mixture of $\beta$ - $O$ - $\beta$-D-glucopyranosyl- $\beta$-sitosterol and $3 \beta$ - $O$ - $\beta$-D-glucopyranosylstigmasterol isolated from stems of Stemodia maritima 


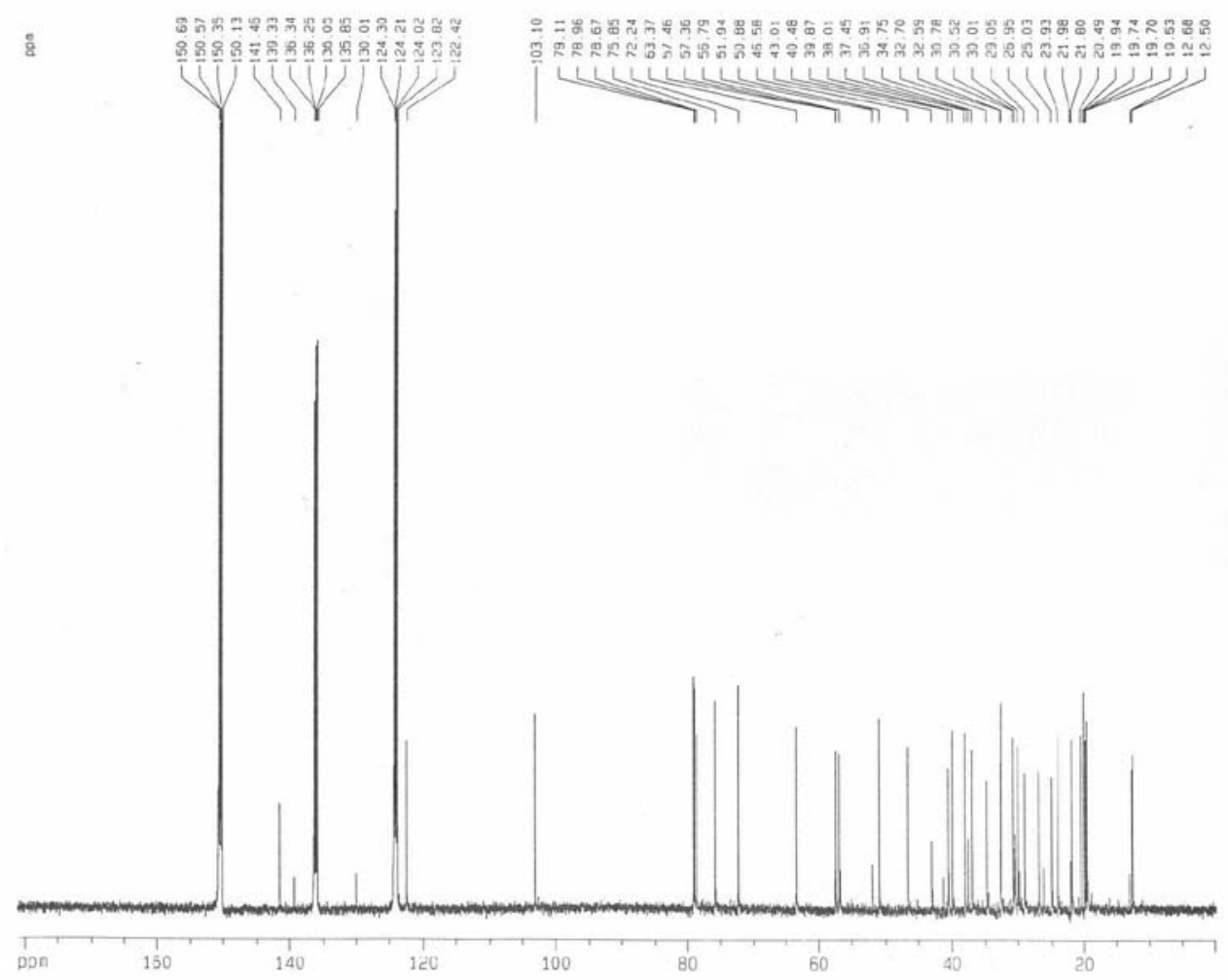

Figure S37. ${ }^{13} \mathrm{C}$ NMR spectrum $\left(\mathrm{C}_{5} \mathrm{D}_{5} \mathrm{~N}, 125 \mathrm{MHz}\right)$ of steroidal mixture of $\beta$ - $O$ - $\beta$-D-glucopyranosyl- $\beta$-sitosterol and $3 \beta$ - $O$ - $\beta$-D-glucopyranosylstigmasterol isolated from stems of Stemodia maritima.

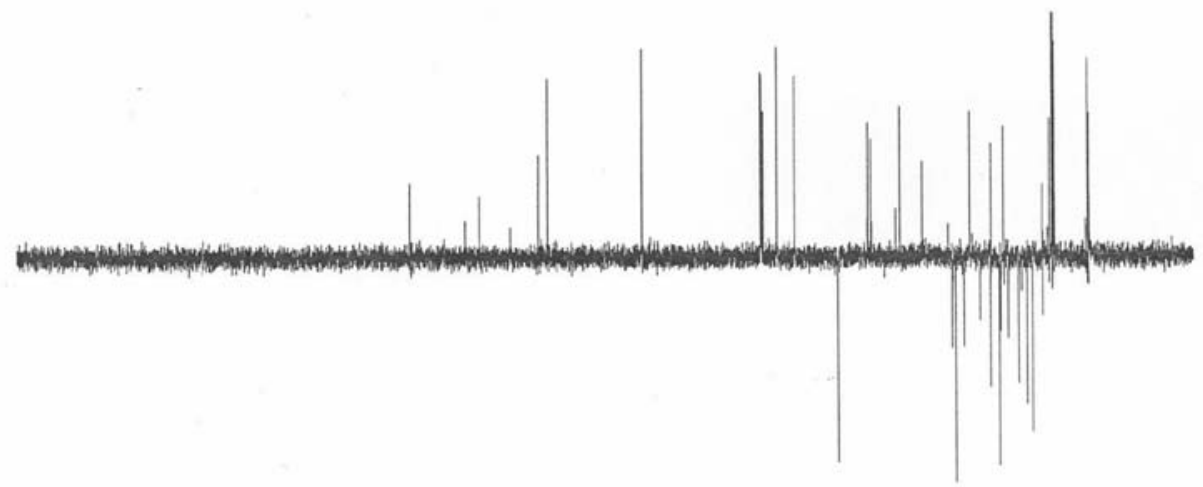




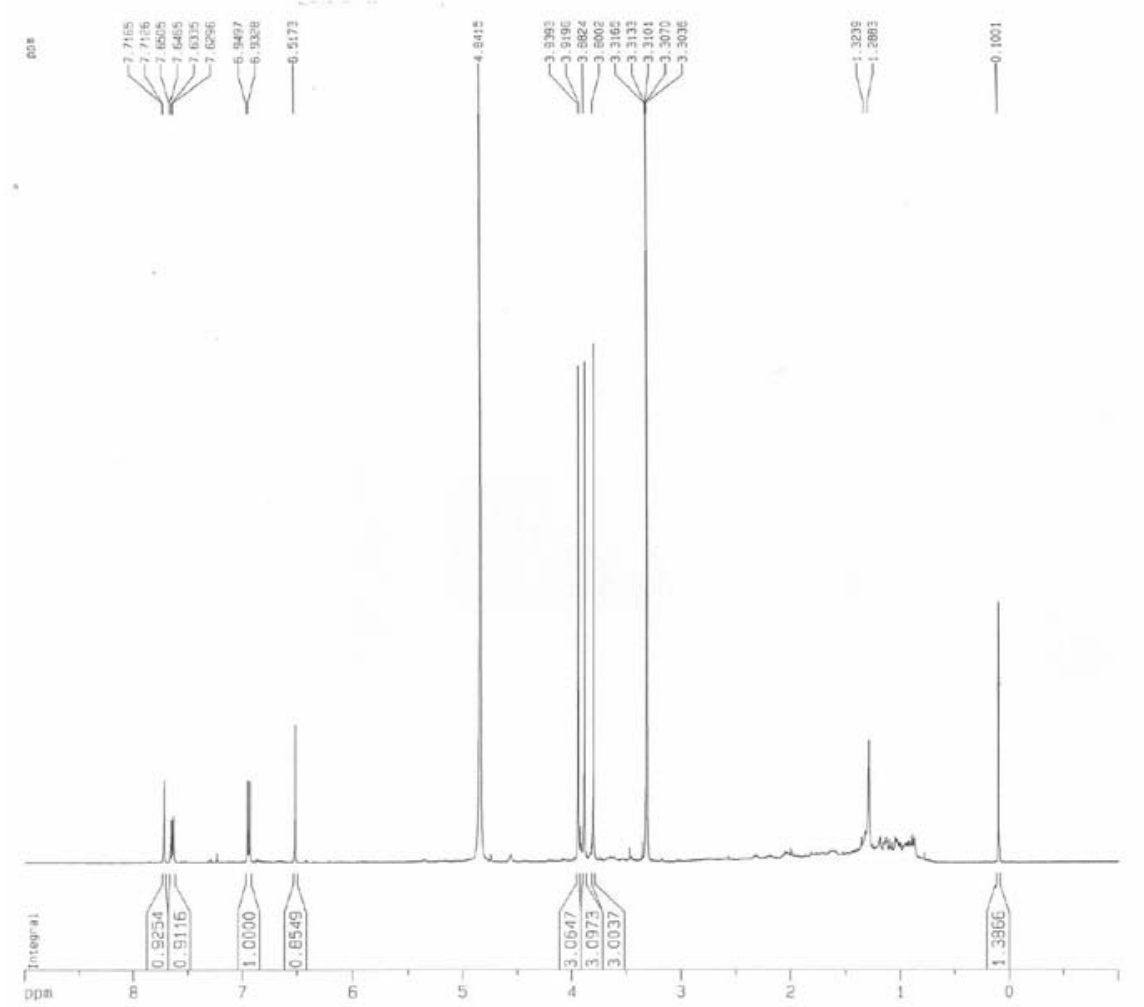

Figure S39. ${ }^{~} \mathrm{H}$ NMR spectrum $\left(\mathrm{CD}_{3} \mathrm{OD}, 500 \mathrm{MHz}\right)$ of 5,7,4'-trihydroxy-3,8,3'-trimethoxyflavone isolated from leaves of Stemodia maritima.

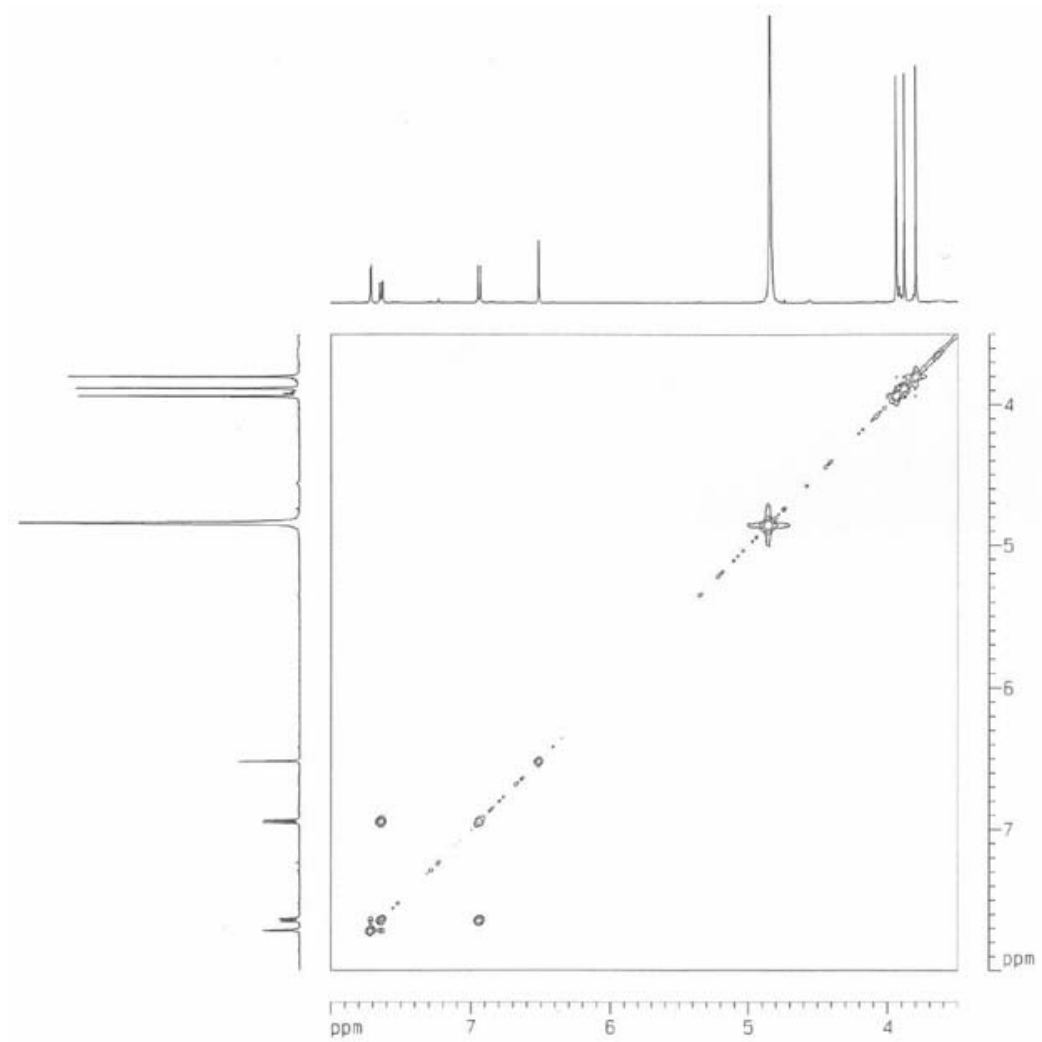

Figure S40. COSY NMR experiment ( $\left.\mathrm{CD}_{3} \mathrm{OD}, 500 \mathrm{MHz}\right)$ of 5,7,4'-trihydroxy-3,8,3'-trimethoxyflavone isolated from leaves of Stemodia maritima. 


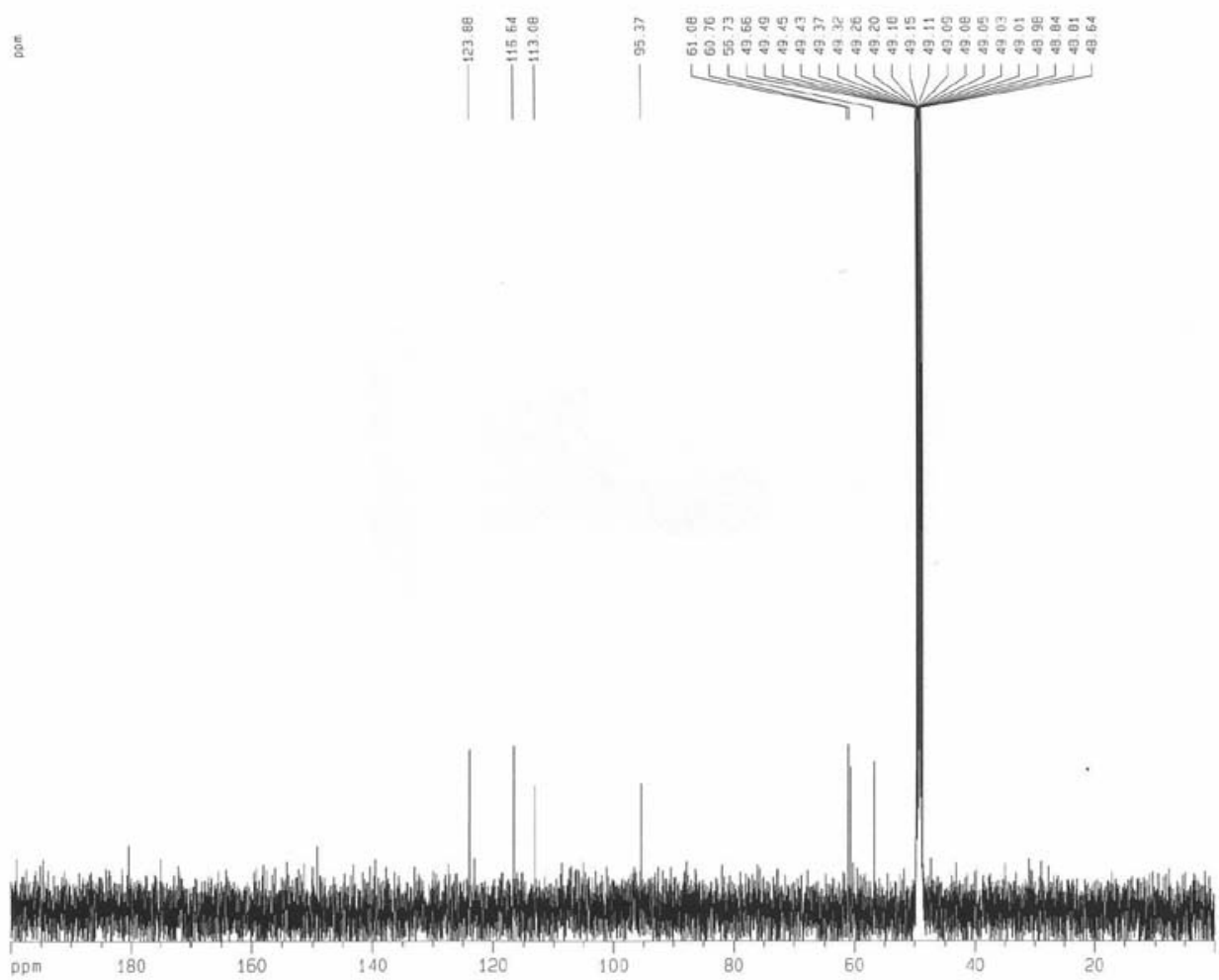

Figure S41. ${ }^{13} \mathrm{C}$ NMR spectrum $\left(\mathrm{CD}_{3} \mathrm{OD}, 125 \mathrm{MHz}\right)$ of 5,7,4'-trihydroxy-3,8,3'-trimethoxyflavone isolated from leaves of Stemodia maritima.

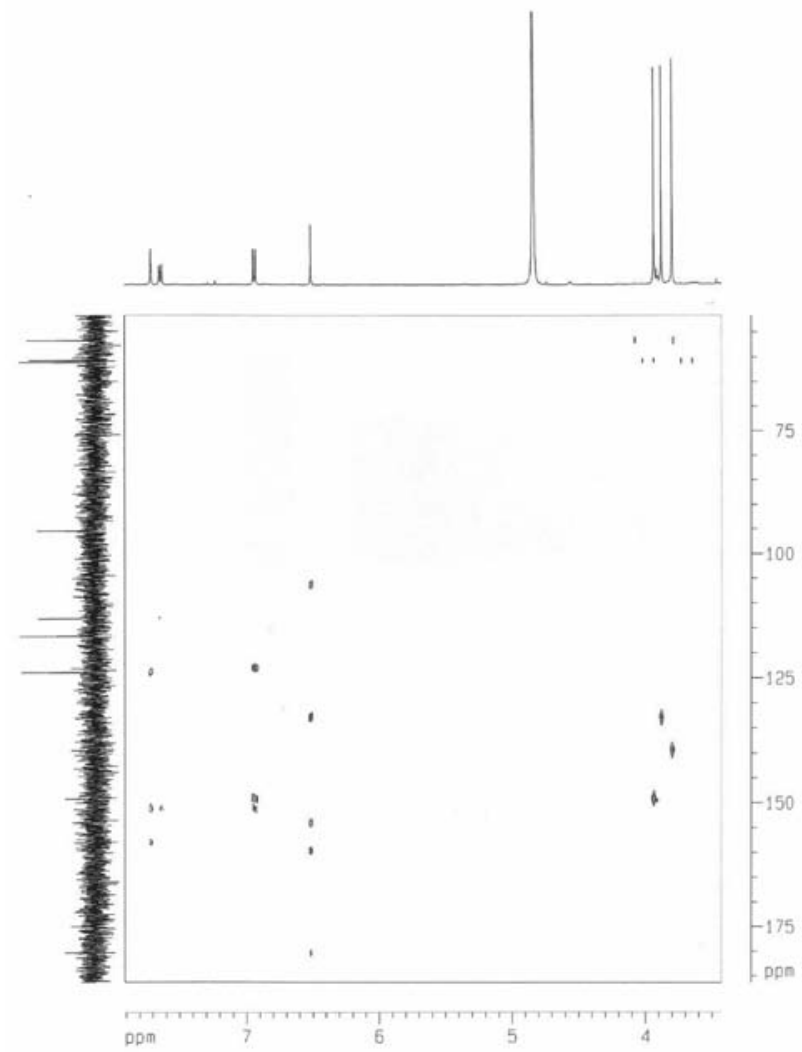

Figure S42. HMBC NMR experiment ( $\left.\mathrm{CD}_{3} \mathrm{OD}, 500 \times 125 \mathrm{MHz}\right)$ of 5,7,4'-trihydroxy-3,8,3'-trimethoxyflavone isolated from leaves of Stemodia maritima. 


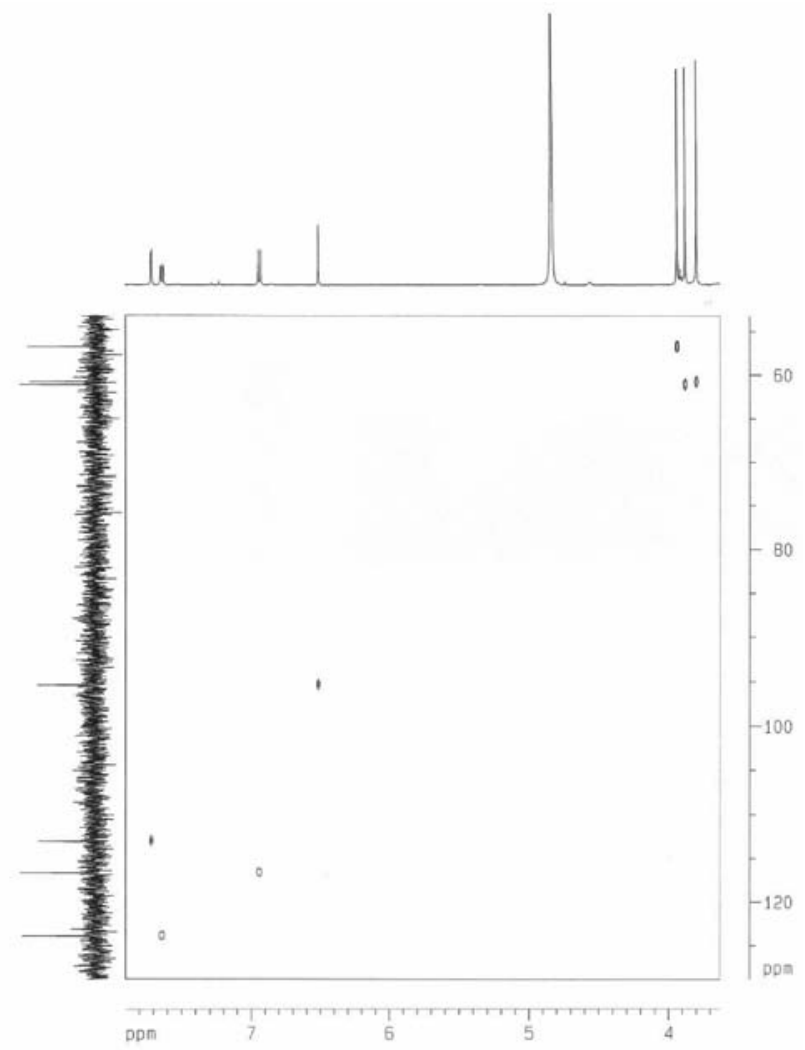

Figure S43. HSQC NMR experiment ( $\left.\mathrm{CD}_{3} \mathrm{OD}, 500 \times 125 \mathrm{MHz}\right)$ of 5,7,4'-trihydroxy-3,8,3'-trimethoxyflavone isolated from leaves of Stemodia maritima. 